\title{
Blocks of Monotone Boolean Functions of Rank 5
}

\author{
Tkachenco V.G. ${ }^{1, *}$, Sinyavsky O.V. ${ }^{2}$ \\ ${ }^{1}$ Institute of Radio, Television, Electronics, Odessa National Academy of Telecommunications named after O. S. Popov, Ukraine \\ ${ }^{2}$ Department of Fundamental Sciences, Odessa Military Academy, Ukraine
}

Copyright $\subset 2016$ by authors, all rights reserved. Authors agree that this article remains permanently open access under the terms of the Creative Commons Attribution License 4.0 international License.

\begin{abstract}
Based on the classification of monotone Boolean functions (MBFs) on the types and the method of building MBFs blocks, an analysis is conducted of the MBFs rank 5. Four matrixes for such MBFs are adduced. It is shown that there are 7581 MBFs rank 5, 276 of them are MBFs of maximal types. These $7581 \mathrm{MBF}$ are contained in 522 blocks or 23 groups of isomorphic blocks or 6 groups of similar blocks. The offered methods can be used to analyze large MBFs ranks. In previous articles were shown how MBFs used in telecommunications for analyzing networks and building codes for cryptosystems.
\end{abstract}

Keywords Monotone Boolean Functions, Disjunctive Complement, Conjunctive Complement, Dedekind Number, Free Distributive Lattice

\section{Introduction}

In $1897 \mathrm{R}$. Dedekind has published article [1] in which the number of elements of a free distributive lattice with four generators has been found. The number $\psi(n)$ of elements of a free distributive lattice with $n$ generators coincides with number of antichains in the unit $n$-dimensional cube. In the language of logic algebra $K(n)=$ $\psi(n)+2$ - the number of the monotone Boolean functions (MBFs), which depend on $n$ variables $x_{1}, \ldots, x_{n}$. The problem of calculating $\psi(n)$ is called Dedekind's problem. $D(0)-D(4)$ computed by $\mathrm{R}$. Dedekind (1897). $D(5)$ are given by Church (1940). $D(6)$ was calculated by Ward (1946), $D(7)$ was calculated by Church (1965) and $D(8)$ by Wiedemann [2] (1991). This is sequence A000372 in Sloane's Online Encyclopedia of Integer Sequences. As it turned out, this problem is quite difficult and cannot be examine through the traditional method of generating functions. At present known ways of calculation $D(n)$. One way of partitioning the number of distinct monotone functions of $n$ variables is to classify them according to the number of distinct input states, at which the function is equal to 1 . Another way of splitting up $D(n)$ is according with the number of conjunctions in the disjunctive normal form [3]. Recent attempts to improve the computational time are e.g. [11, 12]. Recent related work on inequivalent antichains is in [13]. The Dedekind numbers count the number of monotone Boolean function as well as the number of antichains or the number of Sperner families.

In [4-6] for the construction of the new codes for cryptosystems proposed to use a monotone Boolean functions (MBFs). It is necessary to carry out the MBFs analysis methods which are insufficiently developed. In [7] developed a classification of the types of MBFs and enumeration of maximal types of MBFs. Using types of MBFs, constructed cryptosystem based on triangular codes [5]. In [9] developed a method for the construction of MBFs blocks and using this method, as well as method of classification MBFs on types researched all MBFs of ranks from 0 to 4 [8].

However, even for rank 4 has only 168 MBFs, it is not enough for any practical use of the MBFs, nor to establish regularities common to all ranks of MBFs.

These blocks make possible to simplify the calculation of Dedekind numbers. This method is not limited to the number of variables, because for any MBF with any number of variables $n$ using the disjunctive complement and duality can construct a block containing this MBF. For descriptions of all MBFs of $n$ variables it is sufficient to construct only nonisomorphic blocks. This very significantly reduces the description of all MBFs of $n$ variables.

In what follows is an example of calculating for $D(5)$. All MBFs of 5 variables (these MBFs blocks will be described in the following paper) can be divided into 522 blocks, but you can only choose 23 pairwise nonisomorphic. Nonisomorphic blocks are directly related to Dedekind numbers.

The aim of paper is development of a method of the analysis and MBFs classification of rank 5 on the basis of construction of MBFs blocks.

\section{Results}

Consider some of the concepts related to the method of classification MBFs on the types [7]. We say that two 
MBFs of $n$ variables belong to the same type if appropriate this MBFs antichain in a Boolean cube for any $i$ from 0 to $n$ contain the same number of sets with $i$ units. In this case, for each $i$ in minimal disjunctive forms [7] of the MBFs contains the same number of conjunctions, which include $i$ variables. In [7] type of MBFs is defined as a vector $T=$ $\left(a_{0}, a_{1}, \ldots, a_{i}, \ldots, a_{n}\right)$ of $n+1$-th component, which are numbered from left to right from 0 to $n$, where the $i$-th component of the vector $a_{i}$ equal to the number of input sets, the MBFs, containing $i$ units. The number $\mathrm{n}$ is called the rank of a type $T$; the number of non-zero component $v-$ weight of a type $T$; the number $j$ of the first nonzero components on the right - the right border of the $T$; the sum $m$ of all the components of the $T$ - power type $T$. Type $\mathrm{T}$ is called maximal, if an increase of any component by 1 , the resulting vector does not correspond to any type and does not exist MBF corresponding to this vector.

The introduced by us concept of the type of MBF is similar to the concept of the profile of MBF which was introduced in [10]. This concept of the type of MBF is obtained independently and obtained a series of results for him, which had not been obtained for the profile of MBF.

In [8] on the set of MBFs any rank identified three unary operations: duality, conjunctive complement and disjunctive complement. For disjunctive complement $\overline{f_{i}}(n)$ from the $i$-th MBFs $f_{\mathrm{i}}(n)$ must be replaced in minimal disjunctive form each conjunction of $m$ variables on the conjunction of all $n-m$ variables not included in the initial conjunction. For conjunctive complement $f_{i}(n)$ from the $i$-th MBFs $f_{i}(n)$ must be replaced in minimal conjunctive form of each disjunction of $\mathrm{m}$ variables on the disjunction of all $n-m$ variables not included in the initial disjunction. For dual MBFs $f_{i}^{-1}(n)$ from the $i$-th MBFs $f_{i}(n)$ must be replaced in minimal disjunctive form all the operations of conjunction with disjunction operations and simultaneously replace all operations disjunction operations with conjunction operations. In this dual MBF $f_{i}^{-1}(n)$ is resulting in the minimal conjunctive form. For dual MBFs $f_{i}^{-1}(n)$ in the minimal disjunctive form should be received in the form of minimum conjunctive open the brackets and cause similar terms.

Block MBFs rank $n$ is a subset of all MBFs rank $n$, closed with respect to the three operations: duality, conjunctive complement and disjunctive complement. We introduce some definitions. Block power is the number of MBFs which enter into it. Two blocks are similar, if the same power and abstraction from their member of MBFs, these blocks are indistinguishable. Two blocks are isomorphic if any MBFs one block can be resulting from some other MBFs block certain substitution variables. In complement, if both of the MBF to perform one of three operations defined for the block, then the resulting MBF first block is resulting from the resultant MBF another unit of the same substitution variables. By definition isomorphic blocks are similar.

On fig. 1 blocks 1.1 and 1.2 power of 7 MBFs of rank 5 are shown: $a$ ) 1.1 and $b$ ) 1.2 .

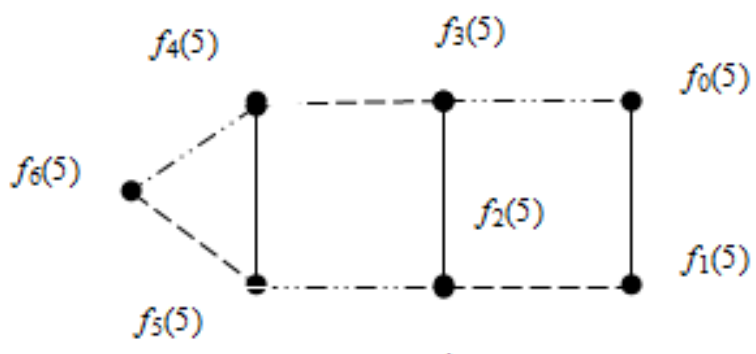

a)

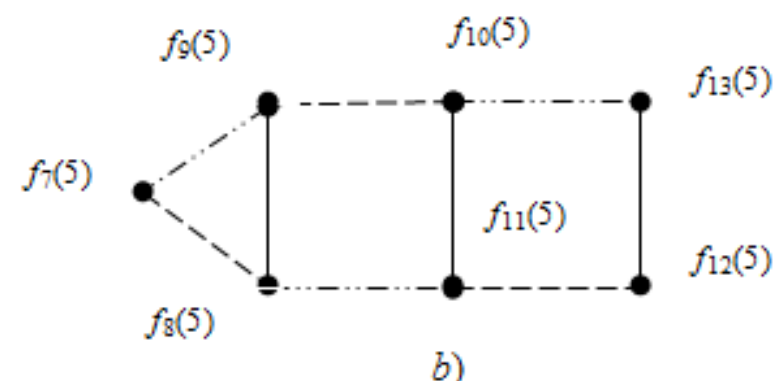

Figure 1. показан ы блок и 1.1 и 1.21 - Blocks of power of 7 MBFs of rank 5: a) 1.1 and b) 1.2

Here $f_{0}(5)$ - zero MBFs (equal 0 at all sets of 5 variables and having type $(0,0,0,0,0,0)), f_{1}(5)-$ unit MBFs (equal 1 at all sets of 5 variables and having type $(1,0,0,0,0,0)), f_{2}(5)=$ $x_{1} x_{2} x_{3} x_{4} x_{5}(0,0,0,0,0,1) ; f_{3}(5)=x_{1} \vee x_{2} \vee x_{3} \vee x_{4} \vee x_{5}$ $(0,5,0,0,0,0) ; f_{4}(5)=x_{1} x_{2} x_{3} x_{4} \vee x_{1} x_{2} x_{3} x_{5} \vee x_{1} x_{2} x_{4} x_{5} \vee x_{1} x_{3} x_{4} x_{5}$ $\vee x_{2} x_{3} x_{4} x_{5}(0,0,0,0,5,0) ; \quad f_{5}(5)=x_{1} x_{2} \vee x_{1} x_{3} \vee x_{1} x_{4} \vee x_{1} x_{5}$ $\vee \vee x_{2} x_{3} x_{2} x_{4} \vee x_{2} x_{5} \vee x_{3} x_{4} \vee x_{3} x_{5} \vee x_{4} x_{5}(0,0,10,0,0,0)$ and $f_{6}(\vee 5)=x_{1} x_{2} x_{3} \vee x_{1} x_{2} x_{4} \vee x_{1} x_{2} x_{5} \vee x_{1} x_{3} x_{4} \vee x_{1} x_{3} x_{5} \vee$ $x_{1} x_{4} x_{5} \vee x_{2} x_{3} x_{4} \vee x_{2} x_{3} x_{5} \vee x_{2} x_{4} x_{5} \vee x_{3} x_{4} x_{5}(0,0,0,10,0,0)$. The block 1.1 contains all MBFs of the maximal types of rank 5 and weight 1 and zero MBFs and unit MBFs. In the block 1.2 we have $f_{7}(5)=x_{1}(0,1,0,0,0,0), f_{8}(5)=x_{2} x_{3} x_{4} x_{5}$ $(0,0,0,0,1,0) ; f_{9}(5)=x_{2} \vee x_{3} \vee x_{4} \vee x_{5}(0,4,0,0,0,0) ; \quad f_{10}(5)=$ $x_{1} x_{2} x_{3} x_{4} \vee x_{1} x_{2} x_{3} x_{5} \vee x_{1} x_{2} x_{4} x_{5} \vee x_{1} x_{3} x_{4} x_{5}(0,0,0,0,4,0) ; \quad f_{11}(5)$ $=x_{1} \vee x_{2} x_{3} \vee x_{2} x_{4} \vee x_{2} x_{5} \vee x_{3} x_{4} \vee x_{3} x_{5} \vee x_{4} x_{5}(0,1,6,0,0,0)$; $f_{12}(5)=x_{1} x_{2} x_{3} \vee x_{1} x_{2} x_{4} \vee x_{1} x_{2} x_{5} \vee x_{1} x_{3} x_{4} \vee x_{1} x_{3} x_{5} \vee x_{1} x_{4} x_{5} \vee$ $x_{2} x_{3} x_{4} x_{5} \quad(0,0,0,6,1,0)$ and $f_{13}(5)=x_{1} x_{2} \vee x_{1} x_{3} \vee x_{1} x_{4} \vee$ $x_{1} x_{5} \vee x_{2} x_{3} x_{4} \vee x_{2} x_{3} x_{5} \vee x_{2} x_{4} x_{5} \vee x_{3} x_{4} x_{5} \quad(0,0,4,4,0,0)$. In the MBFs f11 (5) block $1.2 \ldots f 13$ (5) have the maximal type. The block 1.1 has no isomorphic, and the block 1.2 similar to it has 4 more isomorphic blocks $1.3 \ldots 1.6$ which resulting variable change in $f_{7}(5)$ and use to this MBFs of operations of disjunctive complement and a duality. Thus obtained MBFs $f_{7}(5)-f_{41}(5)$. The third group of 5 isomorphic blocks $1.7 \ldots 1.11$, similar to the block 1.2, resulting if instead of MBFs $f_{7}(5)$ to take MBFs $f_{42}(5)=x_{1} x_{2}$ $\vee x_{1} x_{3} \vee x_{1} x_{4} \vee x_{1} x_{5} \vee x_{2} x_{3} x_{4} x_{5}(0,0,4,0,1,0)$ or one from 4 MBFs, resulting from $f_{42}(5)$ change of variables. In the block 1.7 we have $f_{43}(5)=x_{1} \vee x_{2} x_{3} x_{4} \vee x_{2} x_{3} x_{5} \vee x_{2} x_{4} x_{5} \vee$ $x_{3} x_{4} x_{5}(0,1,0,4,0,0) ; \quad f_{44}(5)=x_{1} x_{2} x_{3} \vee x_{1} x_{2} x_{4} \vee x_{1} x_{2} x_{5} \vee x_{1} x_{3} x_{4}$ $\vee x_{1} x_{3} x_{5} \vee x_{1} x_{4} x_{5}(0,0,0,6,0,0) ; \quad f_{45}(5)=x_{2} x_{3} \vee x_{2} x_{4} \vee x_{2} x_{5}$ $\vee x_{3} x_{4} \vee x_{3} x_{5} \vee x_{4} x_{5}(0,0,6,0,0,0) ; \quad f_{46}(5)=x_{2} x_{3} x_{4} \vee x_{2} x_{3} x_{5}$ $\vee x_{2} x_{4} x_{5} \vee x_{3} x_{4} x_{5}(0,0,0,4,0,0) ; \quad f_{47}(5)=x_{1} x_{2} \vee x_{1} x_{3} \vee x_{1} x_{4}$ 
$\vee x_{1} x_{5}(0,0,4,0,0,0)$ and $f_{48}(5)=x_{1} \vee x_{2} x_{3} x_{4} x_{5}(0,1,0,0,1,0)$. Thus obtained MBFs $f_{42}(5)-f_{76}(5)$. The fourth group of isomorphic blocks $1.12 \ldots 1.21$, similar to the block 1.2 resulting if instead of MBFs $f_{7}(5)$ to take MBFs $f_{77}(5)=x_{1} x_{2}$ $\vee x_{1} x_{3} \vee x_{2} x_{3}(0,0,3,0,0,0)$ or one from 9 MBFs resulting from $f_{77}(5)$ change of variables. Use to $f_{77}(5)$ operations of disjunctive complement and a duality, consistently we receive the block 1.12, in which $f_{78}(5)=x_{1} x_{4} x_{5} \vee x_{2} x_{4} x_{5} \vee$ $x_{3} x_{4} x_{5}(0,0,0,3,0,0) ; \quad f_{79}(5)=x_{4} \vee x_{5} \vee x_{1} x_{2} x_{3}(0,2,0,1,0,0)$; $f_{80}(5)=x_{4} x_{5} \vee x_{1} x_{2} x_{3} x_{4} \vee x_{1} x_{2} x_{3} x_{5}(0,0,1,0,2,0) ; \quad f_{81}(5)=x_{1} x_{4}$ $\vee x_{1} x_{5} \vee x_{2} x_{4} \vee x_{2} x_{5} \vee x_{3} x_{4} \vee x_{3} x_{5} \vee x_{4} x_{5}(0,0,7,0,0,0) ; f_{82}(5)=$ $x_{1} x_{2} x_{3} \vee x_{1} x_{2} x_{4} \vee x_{1} x_{2} x_{5} \vee x_{1} x_{3} x_{4} \vee x_{1} x_{3} x_{5} \vee x_{2} x_{3} x_{4} \vee x_{2} x_{3} x_{5}$ $(0,0,0,7,0,0)$ and $f_{83}(5)=x_{1} x_{2} \vee x_{1} x_{3} \vee x_{2} x_{3} \vee x_{1} x_{4} x_{5} \vee$ $x_{2} x_{4} x_{5} \vee x_{3} x_{4} x_{5} \quad(0,0,3,3,0,0)$. Thus obtained MBFs $f_{77}(5) \ldots$ $f_{146}(5)$. The fifth group of isomorphic blocks $1.22 \ldots 1.31$, similar to the block 1.2 resulting if instead of MBFs $f_{7}(5)$ to take MBFs $f_{147}(5)=x_{1} x_{2} \vee x_{1} x_{3} x_{4} \vee x_{1} x_{3} x_{5} \vee x_{1} x_{4} x_{5} \vee x_{2} x_{3} x_{4} \vee$ $x_{2} x_{3} x_{5} \vee x_{2} x_{4} x_{5}(0,0,1,6,0,0)$ or one from 9 MBFs resulting from $f_{147}(5)$ change of variables. Use to $f_{147}(5)$ operations of disjunctive complement and a duality, consistently we receive the block 1.22, in which $f_{148}(5)=x_{1} x_{3} \vee x_{1} x_{4} \vee x_{1} x_{5} \vee$ $x_{2} x_{3} \vee x_{2} x_{4} \vee x_{2} x_{5} \vee x_{3} x_{4} x_{5}(0,0,6,1,0,0) ; \quad f_{149}(5)=x_{1} x_{2} x_{3} \vee$ $x_{1} x_{2} x_{4} \vee x_{1} x_{2} x_{5} \vee x_{3} x_{4} x_{5}(0,0,0,4,0,0) ; \quad f_{150}(5)=x_{1} x_{2} \vee x_{3} x_{4} \vee$ $x_{3} x_{5} \vee x_{4} x_{5} \quad(0,0,4,0,0,0) ; \quad f_{151}(5)=x_{1} x_{3} x_{4} \vee x_{1} x_{3} x_{5} \vee x_{1} x_{4} x_{5}$ $\vee x_{2} x_{3} x_{4} \vee x_{2} x_{3} x_{5} \vee x_{2} x_{4} x_{5}(0,0,0,6,0,0) ; f_{152}(5)=x_{1} x_{3} \vee x_{1} x_{4}$ $\vee x_{1} x_{5} \vee x_{2} x_{3} \vee x_{2} x_{4} \vee x_{2} x_{5}(0,0,6,0,0,0)$ and $f_{153}(5)=x_{1} x_{2}$ $\checkmark x_{3} x_{4} x_{5} \quad(0,0,1,1,0,0)$. Thus obtained MBFs $f_{147}(5) \ldots f_{216}(5)$. The sixth group of isomorphic blocks $1.32 \ldots 1.51$, similar to the block 1.2 resulting if instead of MBFs $f_{7}(5)$ to take MBFs $f_{217}(5)=x_{1} x_{2} \vee x_{1} x_{3} \vee x_{1} x_{4} \vee x_{2} x_{3} x_{4}$ $(0,0,3,1,0,0)$ or one from 19 MBFs resulting from $f_{217}(5)$ change of variables. Use to $f_{217}(5)$ operations of disjunctive complement and a duality, consistently we receive the block 1.32, in which $f_{218}(5)=x_{1} x_{5} \vee x_{2} x_{3} x_{5} \vee x_{2} x_{4} x_{5} \vee x_{3} x_{4} x_{5}$ $(0,0,1,3,0,0) ; \quad f_{219}(5)=x_{5} \vee x_{1} x_{2} x_{3} \vee x_{1} x_{2} x_{4} \vee x_{1} x_{3} x_{4}$ $(0,1,0,3,0,0) ; \quad f_{220}(5)=x_{2} x_{5} x_{3} x_{5} \vee x_{4} x_{5} \vee x_{1} x_{2} x_{3} x_{4}$ $(0,0,3,0,1,0) ; \quad f_{221}(5)=x_{1} x_{5} \vee x_{2} x_{5} \vee x_{3} x_{5} \vee x_{4} x_{5} \vee x_{2} x_{3} x_{4}$ $(0,0,4,1,0,0) ; \quad f_{222}(5)=x_{1} x_{5} \vee x_{1} x_{2} x_{3} \vee x_{1} x_{2} x_{4} \vee x_{1} x_{3} x_{4} \vee$ $x_{2} x_{3} x_{4} \quad(0,0,1,4,0,0)$ and $f_{223}(5)=x_{1} x_{2} \vee x_{1} x_{3} \vee x_{1} x_{4} \vee$ $x_{2} x_{3} x_{5} \vee x_{2} x_{4} x_{5} \vee x_{3} x_{4} x_{5} \quad(0,0,3,3,0,0)$. Thus obtained MBFs $f_{217}(5) \ldots f_{356}(5)$. The seventh group of isomorphic blocks $1.52 \ldots 1.81$, similar to the block 1.2 resulting if instead of MBFs $f_{7}(5)$ to take MBFs $f_{357}(5)=x_{1} x_{2} \vee x_{1} x_{3} \vee x_{1} x_{4} x_{5} \vee$ $x_{2} x_{3} x_{4} \vee x_{2} x_{3} x_{5}(0,0,2,3,0,0)$ or one from 29 MBFs resulting from $f_{357}(5)$ change of variables. Use to $f_{357}(5)$ operations of disjunctive complement and a duality, consistently we receive the block 1.52, in $f_{358}(5)=x_{1} x_{4} \vee x_{1} x_{5} \vee x_{2} x_{3} \vee x_{2} x_{4} x_{5}$ $\vee x_{3} x_{4} x_{5}(0,0,3,2,0,0) ; \quad f_{359}(5)=x_{1} x_{2} x_{3} \vee x_{1} x_{2} x_{4} \vee x_{1} x_{2} x_{5} \vee$ $x_{1} x_{3} x_{4} \vee x_{1} x_{3} x_{5} \vee x_{2} x_{4} x_{5} \vee x_{3} x_{4} x_{5}(0,0,0,7,0,0) ; f_{360}(5)=x_{1} x_{2} \vee$ $x_{1} x_{3} \vee x_{2} x_{4} \vee x_{2} x_{5} \vee x_{3} x_{4} \vee x_{3} x_{5} \vee x_{4} x_{5} \quad(0,0,7,0,0,0) ; \quad f_{361}(5)$ $=x_{1} x_{4} x_{5} \vee x_{2} x_{3} x_{4} \vee x_{2} x_{3} x_{5} \quad(0,0,0,3,0,0) ; \quad f_{362}(5)=x_{1} x_{4} \vee$ $x_{1} x_{5} \vee x_{2} x_{3}(0,0,3,0,0,0)$ and $f_{363}(5)=x_{1} x_{2} \vee x_{1} x_{3} \vee x_{2} x_{4} x_{5}$ $\vee x_{3} x_{4} x_{5}(0,0,2,2,0,0)$. Thus obtained MBFs $f_{357}(5) \ldots f_{566}(5)$. In total for MBFs 5 of a rank there are 81 similar blocks of power 7. They contain 567 MBFs. In each of these blocks there is 1 self-dual MBFs, 1 disjunctive selfcomplementary MBFs and 1 conjunctive self- complementary MBFs.

On fig. 2 MBFs blocks of rank 5 are shown: a) 2.1 power 4 and $b) 3.1$ power 6 :
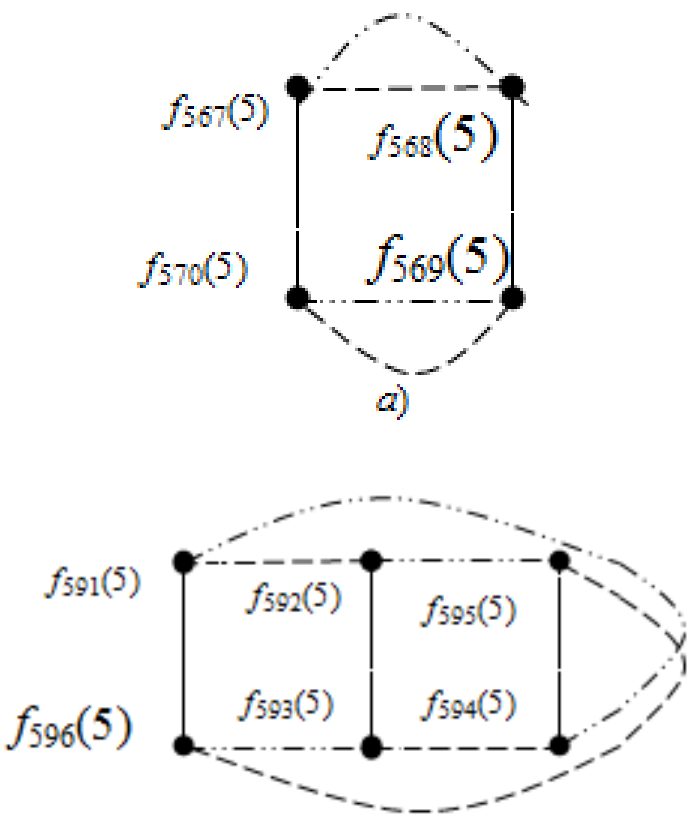

b)

Figure 2. MBFs Blocks of rank 5: a) 2.1 and b) 3.1

In the block 2.1 we have $f_{567}(5)=x_{1} x_{2} \vee x_{1} x_{5} \vee x_{2} x_{3} \vee x_{3} x_{4}$ $\vee x_{4} x_{5}(0,0,5,0,0,0) ; f_{568}(5)=x_{1} x_{2} x_{3} \vee x_{1} x_{2} x_{5} \vee x_{1} x_{4} x_{5} \vee x_{2} x_{3} x_{4}$ $\vee x_{3} x_{4} x_{5}(0,0,0,5,0,0) ; f_{569}(5)=x_{1} x_{3} \vee x_{1} x_{4} \vee x_{2} x_{4} \vee x_{2} x_{5} \vee x_{3} x_{5}$ $(0,0,5,0,0,0)$ and $f_{570}(5)=x_{1} x_{2} x_{4} \vee x_{1} x_{3} x_{4} \vee x_{1} x_{3} x_{5} \vee x_{2} x_{3} x_{5}$ $\vee x_{2} x_{4} x_{5}(0,0,0,5,0,0)$. In total for MBFs 5 of a rank there are 6 isomorphic blocks of power 4 . They contain $24 \mathrm{MBFs}$ $f_{567}(5) \ldots f_{590}(5)$. In the block 3.1 we have $f_{591}(5)=x_{1} x_{2} \vee x_{3} x_{4}$ $\vee x_{1} x_{3} x_{5} \vee x_{2} x_{4} x_{5}(0,0,2,2,0,0) ; \quad f_{592}(5)=x_{1} x_{3} \vee x_{2} x_{4} \vee x_{1} x_{2} x_{5}$ $\vee x_{3} x_{4} x_{5}(0,0,2,2,0,0) ; f_{593}(5)=x_{1} x_{4} \vee x_{2} x_{3} \vee x_{1} x_{2} x_{5} \vee x_{3} x_{4} x_{5}$ $(0,0,2,2,0,0) ; \quad f_{594}(5)=x_{1} x_{2} \vee x_{3} x_{4} \vee x_{1} x_{4} x_{5} \vee x_{2} x_{3} x_{5}$ $(0,0,2,2,0,0) ; \quad f_{595}(5)=x_{1} x_{3} \vee x_{2} x_{4} \vee x_{1} x_{4} x_{5} \vee x_{2} x_{3} x_{5}$ $(0,0,2,2,0,0)$ and $f_{596}(5)=x_{1} x_{4} \vee x_{2} x_{3} \vee x_{1} x_{3} x_{5} \vee x_{2} x_{4} x_{5}$ $(0,0,2,2,0,0)$. In total for MBFs 5 of a rank there are 5 isomorphic blocks of power 6 . They contain $30 \mathrm{MBFs}$ $f_{591}(5) \ldots f_{620}(5)$.

On fig. 3 block 4.1 power of $14 \mathrm{MBFs}$ of rank 5 is shown.

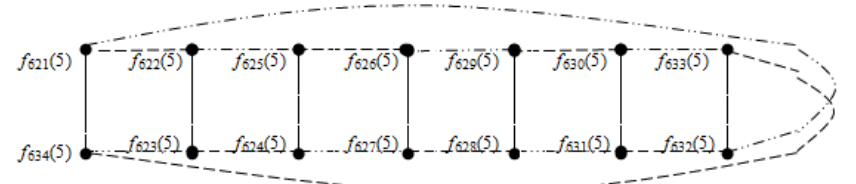

Figure 3 Block 4.1 power of $14 \mathrm{MBFs}$ of rank 5

There are 390 blocks, similar to block 4.1. These blocks can be divided into 12 groups, each of which consists of isomorphic between blocks. The first group consists of 10 blocks 4.1 .. 4.10. In block 4.1 we have: 1) f621(5) $=\mathrm{x} 1 \mathrm{x} 2(0,0,1,0,0,0) ; 2) \mathrm{f} 622(5)=\mathrm{x} 3 \mathrm{x} 4 \mathrm{x} 5(0,0,0,1,0,0) ; 3)$ 
$\mathrm{f} 623(5)=x 3 \vee x 4 \vee x 5(0,3,0,0,0,0) ; 4) \mathrm{f} 624(5)=x 1 \times 2 \times 3 \times 4$ $\vee x 1 \times 2 x 3 \times 5 \vee x 1 \times 2 x 4 \times 5(0,0,0,0,3,0) ; 5) \mathrm{f} 625(5)=x 1 \vee x 2$ $\vee \times 3 x 4 \vee x 3 \times 5 \vee x 4 \times 5(0,2,3,0,0,0) ; 6)$ f626(5) $=x 1 \times 2 \times 3 \vee$ $\mathrm{x} 1 \mathrm{x} 2 \mathrm{x} 4 \vee \mathrm{x} 1 \mathrm{x} 2 \mathrm{x} 5 \vee \mathrm{x} 1 \mathrm{x} 3 \mathrm{x} 4 \mathrm{x} 5 \vee \mathrm{x} 2 \mathrm{x} 3 \mathrm{x} 4 \mathrm{x} 5(0,0,0,3,2,0) ; 7)$ $\mathrm{f} 627(5)=\mathrm{x} 1 \mathrm{x} 2 \vee \mathrm{x} 1 \mathrm{x} 3 \vee \mathrm{x} 1 \mathrm{x} 4 \vee \mathrm{x} 1 \mathrm{x} 5 \vee \mathrm{x} 2 \mathrm{x} 3 \vee \mathrm{x} 2 \mathrm{x} 4 \vee$ $\mathrm{x} 2 \mathrm{x} 5 \vee \mathrm{x} 3 \mathrm{x} 4 \mathrm{x} 5(0,0,7,1,0,0) ; 8) \mathrm{f} 628(5)=\mathrm{x} 1 \mathrm{x} 2 \vee \mathrm{x} 1 \times 3 \times 4 \vee$ $\mathrm{x} 1 \mathrm{x} 3 \mathrm{x} 5 \vee \mathrm{x} 1 \mathrm{x} 4 \mathrm{x} 5 \vee \mathrm{x} 2 \mathrm{x} 3 \mathrm{x} 4 \vee \mathrm{x} 2 \mathrm{x} 3 \mathrm{x} 5 \vee \mathrm{x} 2 \mathrm{x} 4 \mathrm{x} 5 \vee \mathrm{x} 3 \mathrm{x} 4 \mathrm{x} 5$ $(0,0,1,7,0,0) ; 9) \mathrm{f} 629(5)=\mathrm{x} 1 \mathrm{x} 2 \mathrm{x} 3 \vee \mathrm{x} 1 \mathrm{x} 2 \mathrm{x} 4 \vee \mathrm{x} 1 \mathrm{x} 2 \mathrm{x} 5 \vee$ $\mathrm{x} 1 \mathrm{x} 3 \mathrm{x} 4 \vee \mathrm{x} 1 \mathrm{x} 3 \mathrm{x} 5 \vee \mathrm{x} 1 \mathrm{x} 4 \mathrm{x} 5 \vee \mathrm{x} 2 \mathrm{x} 3 \mathrm{x} 4 \vee \mathrm{x} 2 \mathrm{x} 3 \mathrm{x} 5 \vee \mathrm{x} 2 \mathrm{x} 4 \mathrm{x} 5$ $(0,0,0,9,0,0) ; 10) \mathrm{f} 630(5)=\mathrm{x} 1 \mathrm{x} 3 \vee \mathrm{x} 1 \mathrm{x} 4 \vee \mathrm{x} 1 \mathrm{x} 5 \vee \mathrm{x} 2 \mathrm{x} 3 \vee$ $\mathrm{x} 2 \mathrm{x} 4 \vee \mathrm{x} 2 \mathrm{x} 5 \vee \mathrm{x} 3 \mathrm{x} 4 \vee \mathrm{x} 3 \mathrm{x} 5 \vee \mathrm{x} 4 \mathrm{x} 5(0,0,9,0,0,0) ; 11) \mathrm{f} 631(5)$ $=\mathrm{x} 3 \mathrm{x} 4 \mathrm{x} 5 \vee \mathrm{x} 1 \mathrm{x} 2 \mathrm{x} 3 \mathrm{x} 4 \vee \mathrm{x} 1 \mathrm{x} 2 \mathrm{x} 3 \mathrm{x} 5 \vee \mathrm{x} 1 \mathrm{x} 2 \mathrm{x} 4 \mathrm{x} 5(0,0,0,1,3,0)$; 12) $\mathrm{f} 632(5)=x 3 \vee x 4 \vee x 5 \vee x 1 \times 2(0,3,1,0,0,0)$; 13) f633(5) $=x 1 \times 3 \times 4 \times 5 \vee x 2 \times 3 \times 4 \times 5(0,0,0,0,2,0) ; 14) \mathrm{f} 634(5)=x 1 \vee x 2$ $(0,2,0,0,0,0)$. The first group contains 140 MBFs f621(5)...f760(5). The second group consists of 15 blocks $4.11 \ldots 4.25$. In the block 4.11 we have: 1) $\mathrm{f} 761(5)=\mathrm{x} 1 \mathrm{x} 2$ $\vee \times 3 \times 4 \quad(0,0,2,0,0,0) ; 2) \quad \mathrm{f} 762(5)=\mathrm{x} 1 \times 2 \times 5 \vee \times 3 \times 4 \times 5$ $(0,0,0,2,0,0) ; 3) \mathrm{f} 763(5)=\mathrm{x} 5 \vee \mathrm{x} 1 \mathrm{x} 3 \vee \mathrm{x} 1 \mathrm{x} 4 \vee \mathrm{x} 2 \mathrm{x} 3 \vee \mathrm{x} 2 \mathrm{x} 4$ $(0,1,4,0,0,0) ; 4) \mathrm{f} 764(5)=\mathrm{x} 1 \times 3 \times 5 \vee \times 1 \times 4 \times 5 \vee \times 2 \times 3 \times 5 \vee$ $\mathrm{x} 2 \mathrm{x} 4 \mathrm{x} 5 \vee \mathrm{x} 1 \mathrm{x} 2 \mathrm{x} 3 \mathrm{x} 4(0,0,0,4,1,0) ; 5) \mathrm{f} 765(5)=\mathrm{x} 1 \mathrm{x} 2 \vee \mathrm{x} 1 \mathrm{x} 5$ $\vee \mathrm{x} 2 \mathrm{x} 5 \vee \mathrm{x} 3 \mathrm{x} 4 \vee \mathrm{x} 3 \times 5 \vee \mathrm{x} 4 \mathrm{x} 5(0,0,6,0,0,0) ; 6) \mathrm{f} 766(5)=$ $\mathrm{x} 1 \mathrm{x} 2 \mathrm{x} 3 \vee \mathrm{x} 1 \mathrm{x} 2 \mathrm{x} 4 \vee \mathrm{x} 1 \mathrm{x} 2 \mathrm{x} 5 \vee \mathrm{x} 1 \mathrm{x} 3 \mathrm{x} 4 \vee \mathrm{x} 2 \mathrm{x} 3 \mathrm{x} 4 \vee$ $\mathrm{x} 3 \mathrm{x} 4 \mathrm{x} 5(0,0,0,6,0,0) ; 7) \mathrm{f} 767(5)=\mathrm{x} 1 \mathrm{x} 3 \vee \mathrm{x} 1 \mathrm{x} 4 \vee \mathrm{x} 2 \mathrm{x} 3 \vee$ $\mathrm{x} 2 \mathrm{x} 4 \vee \mathrm{x} 1 \mathrm{x} 2 \mathrm{x} 5 \vee \mathrm{x} 3 \mathrm{x} 4 \mathrm{x} 5(0,0,4,2,0,0) ; 8) \mathrm{f} 768(5)=\mathrm{x} 1 \mathrm{x} 2 \vee$ $\mathrm{x} 3 \mathrm{x} 4 \vee \mathrm{x} 1 \mathrm{x} 3 \mathrm{x} 5 \vee \mathrm{x} 1 \mathrm{x} 4 \mathrm{x} 5 \vee \mathrm{x} 2 \mathrm{x} 3 \mathrm{x} 5 \vee \mathrm{x} 2 \mathrm{x} 4 \mathrm{x} 5(0,0,2,4,0,0) ; 9)$ $\mathrm{f} 769(5)=\mathrm{x} 1 \mathrm{x} 2 \mathrm{x} 3 \vee \mathrm{x} 1 \mathrm{x} 2 \mathrm{x} 4 \vee \mathrm{x} 1 \mathrm{x} 3 \mathrm{x} 4 \vee \mathrm{x} 1 \mathrm{x} 3 \mathrm{x} 5 \vee \mathrm{x} 1 \mathrm{x} 4 \mathrm{x} 5 \vee$ $\mathrm{x} 2 \mathrm{x} 3 \mathrm{x} 4 \vee \mathrm{x} 2 \mathrm{x} 3 \mathrm{x} 5 \vee \mathrm{x} 2 \mathrm{x} 4 \mathrm{x} 5 \quad(0,0,0,8,0,0) ; 10) \mathrm{f} 770(5)=$ $\mathrm{x} 1 \mathrm{x} 3 \vee \mathrm{x} 1 \mathrm{x} 4 \vee \mathrm{x} 1 \mathrm{x} 5 \vee \mathrm{x} 2 \mathrm{x} 3 \vee \mathrm{x} 2 \mathrm{x} 4 \vee \mathrm{x} 2 \mathrm{x} 5 \vee \mathrm{x} 3 \mathrm{x} 5 \vee \mathrm{x} 4 \mathrm{x} 5$ $(0,0,8,0,0,0) ; 11) \mathrm{f} 771(5)=\mathrm{x} 1 \times 2 \times 5 \vee \times 3 \times 4 \times 5 \vee \times 1 \times 2 \times 3 \times 4$ $(0,0,0,2,1,0) ; 12) \mathrm{f} 772(5)=x 5 \vee \times 1 \times 2 \vee \times 3 \times 4 \quad(0,1,2,0,0,0)$; 13) $\mathrm{f} 773(5)=x 1 \times 3 \times 5 \vee x 1 \times 4 \times 5 \vee x 2 \times 3 \times 5 \vee x 2 \times 4 \times 5$ $(0,0,0,4,0,0) ; 14) \mathrm{f} 774(5)=\mathrm{x} 1 \mathrm{x} 3 \vee \mathrm{x} 1 \mathrm{x} 4 \vee \mathrm{x} 2 \mathrm{x} 3 \vee \mathrm{x} 2 \mathrm{x} 4$ $(0,0,4,0,0,0)$. The second group contains 210 MBFs f761(5)..f970(5). The third group consists of 15 blocks $4.26 \ldots 4.40$. In the block 4.26 we have: 1) $\mathrm{f} 971(5)=\mathrm{x} 1 \mathrm{x} 2$ $\vee x 1 \times 3 \vee x 2 x 3 \vee x 3 x 4 \times 5 \quad(0,0,3,1,0,0) ; 2) f 972(5)=x 1 \times 2$ $\vee \mathrm{x} 1 \mathrm{x} 4 \mathrm{x} 5 \vee \mathrm{x} 2 \mathrm{x} 4 \mathrm{x} 5 \vee \mathrm{x} 3 \mathrm{x} 4 \mathrm{x} 5(0,0,1,3,0,0) ; 3) \mathrm{f} 973(5)=$ $\mathrm{x} 1 \mathrm{x} 4 \vee \mathrm{x} 1 \mathrm{x} 5 \vee \mathrm{x} 2 \mathrm{x} 4 \vee \mathrm{x} 2 \mathrm{x} 5 \vee \mathrm{x} 1 \mathrm{x} 2 \mathrm{x} 3 \quad(0,0,4,1,0,0) ; \quad 4)$ $\mathrm{f} 974(5)=\mathrm{x} 4 \mathrm{x} 5 \vee \mathrm{x} 1 \mathrm{x} 3 \mathrm{x} 4 \vee \mathrm{x} 1 \mathrm{x} 3 \mathrm{x} 5 \vee \mathrm{x} 2 \mathrm{x} 3 \mathrm{x} 4 \vee \mathrm{x} 2 \mathrm{x} 3 \mathrm{x} 5$ $(0,0,1,4,0,0) ; 5) \mathrm{f} 975(5)=\mathrm{x} 3 \mathrm{x} 4 \vee \mathrm{x} 3 \mathrm{x} 5 \vee \mathrm{x} 4 \mathrm{x} 5 \vee \mathrm{x} 1 \mathrm{x} 2 \mathrm{x} 4$ $\vee x 1 \times 2 \times 5(0,0,3,2,0,0) ; 6) \mathrm{f} 976(5)=x 3 \times 4 \vee \times 3 \times 5 \vee x 1 \times 2 \times 3$ $\vee x 1 \times 2 x 4 \vee x 1 x 2 x 5(0,0,2,3,0,0) ; 7)$ f977(5) $=x 1 \times 3 \vee x 2 x 3$ $\vee \mathrm{x} 1 \mathrm{x} 4 \mathrm{x} 5 \vee \mathrm{x} 2 \mathrm{x} 4 \mathrm{x} 5 \vee \mathrm{x} 3 \mathrm{x} 4 \mathrm{x} 5(0,0,2,3,0,0) ; 8) \mathrm{f978}(5)=$ $\left.\mathrm{x} 1 \mathrm{x} 2 \vee \mathrm{x} 1 \mathrm{x} 3 \vee \mathrm{x} 2 \mathrm{x} 3 \vee \mathrm{x} 1 \mathrm{x}_{4} x_{5} \vee x_{2} x_{4} x_{5}(0,0,3,2,0,0) ; 9\right) f_{979}(5)$ $\left.=x_{1} x_{2} \vee x_{1} x_{3} x_{4} \vee x_{1} x_{3} x_{5} \vee x_{2} x_{3} x_{4} \vee x_{2} x_{3} x_{5}(0,0,1,4,0,0) ; 10\right)$ $\left.f_{980}(5)=x_{1} x_{4} \vee x_{1} x_{5} \vee x_{2} x_{4} \vee x_{2} x_{5} \vee x_{3} x_{4} x_{5}(0,0,4,1,0,0) ; 11\right)$ $\left.f_{981}(5)=x_{4} x_{5} \vee x_{1} x_{2} x_{3} \vee x_{1} x_{2} x_{4} \vee x_{1} x_{2} x_{5}(0,0,1,3,0,0) ; 12\right) f_{982}(5)$ $\left.=x_{3} x_{4} \vee x_{3} x_{5} \vee x_{4} x_{5} \vee x_{1} x_{2} x_{3} \quad(0,0,3,1,0,0) ; 13\right) f_{983}(5)=x_{3} x_{4} \vee$ $x_{3} x_{5} \vee x_{1} x_{4} x_{5} \vee x_{2} x_{4} x_{5}(0,0,2,2,0,0)$; 14) $f_{984}(5)=x_{1} x_{3} \vee x_{2} x_{3} \vee$ $x_{1} x_{2} x_{4} \vee x_{1} x_{2} x_{5}(0,0,2,2,0,0)$. The third group contains 210 MBFs $f_{971}(5) \ldots f_{1180}(5)$. The fourth group consists of 20 blocks $4.41 \ldots 4.60$. In the block 4.41 we have: 1) $f_{1181}(5)=$ $\left.x_{1} x_{2} \vee x_{1} x_{3} \vee x_{1} x_{4} \quad(0,0,3,0,0,0) ; 2\right) f_{1182}(5)=x_{2} x_{3} x_{5} \vee x_{2} x_{4} x_{5}$ $\vee x_{3} x_{4} x_{5}(0,0,0,3,0,0)$; 3) $f_{1183}(5)=x_{5} \vee x_{2} x_{3} \vee x_{2} x_{4} \vee x_{3} x_{4}$ $(0,1,3,0,0,0)$; 4) $f_{1184}(5)=x_{1} x_{2} x_{5} \vee x_{1} x_{3} x_{5} \vee x_{1} x_{4} x_{5 . .} x_{1} x_{2} x_{3} x_{4}$
$(0,0,0,3,1,0)$; 5) $f_{1185}(5)=x_{1} \vee x_{2} x_{5} \vee x_{3} x_{5} \vee x_{4} x_{5} \vee x_{2} x_{3} x_{4}$ $(0,1,3,1,0,0)$; 6) $f_{1186}(5)=x_{1} x_{5} \vee x_{1} x_{2} x_{3} \vee x_{1} x_{2} x_{4} \vee x_{1} x_{3} x_{4} \vee$ $x_{2} x_{3} x_{4} x_{5}(0,0,1,3,1,0)$; 7) $f_{1187}(5)=x_{1} x_{2} \vee x_{1} x_{3} \vee x_{1} x_{4} \vee x_{1} x_{5} \vee$ $\left.x_{2} x_{3} x_{5} \vee x_{2} x_{4} x_{5} \vee x_{3} x_{4} x_{5}(0,0,4,3,0,0) ; 8\right) f_{1188}(5)=x_{1} x_{2} \vee x_{1} x_{3}$ $\left.\vee x_{1} x_{4} \vee x_{2} x_{3} x_{4} \vee x_{2} x_{3} x_{5} \vee x_{2} x_{4} x_{5} \vee x_{3} x_{4} x_{5} \quad(0,0,3,4,0,0) ; 9\right)$ $f_{1189}(5)=x_{1} x_{2} x_{3} \vee x_{1} x_{2} x_{4} \vee x_{1} x_{2} x_{5} \vee x_{1} x_{3} x_{4} \vee x_{1} x_{3} x_{5} \vee x_{1} x_{4} x_{5} \vee$ $x_{2} x_{3} x_{4}(0,0,0,7,0,0)$; 10) $f_{1190}(5)=x_{1} x_{5} \vee x_{2} x_{3} \vee x_{2} x_{4} \vee x_{2} x_{5} \vee$ $x_{3} x_{4} \vee x_{3} x_{5} \vee x_{4} x_{5}(0,0,7,0,0,0)$; 11) $f_{1191}(5)=x_{2} x_{3} x_{5} \vee x_{2} x_{4} x_{5} \vee$ $\left.x_{3} x_{4} x_{5} \vee x_{1} x_{2} x_{3} x_{4}(0,0,0,3,1,0) ; 12\right) f_{1192}(5)=x_{5} \vee x_{1} x_{2} \vee x_{1} x_{3}$ $\left.\vee x_{1} x_{4} \quad(0,1,3,0,0,0) ; 13\right) \quad f_{1193}(5)=x_{1} x_{5} \vee x_{2} x_{3} x_{4} x_{5}$ $(0,0,1,0,1,0) ; 14) f_{1194}(5)=x_{1} \vee x_{2} x_{3} x_{4} \quad(0,1,0,1,0,0)$. The fourth group contains $280 \mathrm{MBFs} f_{1181}(5) \ldots f_{1460}(5)$. The fifth group consists of 30 blocks $4.61 \ldots 4.90$. In the block 4.61 we have: 1) $f_{1461}(5)=x_{1} x_{2} \vee x_{1} x_{3} \quad(0,0,2,0,0,0)$; 2) $f_{1462}(5)$ $=x_{2} x_{4} x_{5} \vee x_{3} x_{4} x_{5} \quad(0,0,0,2,0,0)$; 3) $f_{1463}(5)=x_{4} \vee x_{5} \vee x_{2} x_{3}$ $(0,2,1,0,0,0)$; 4) $f_{1464}(5)=x_{1} x_{4} x_{5} \vee x_{1} x_{2} x_{3} x_{4} \vee x_{1} x_{2} x_{3} x_{5}$ $(0,0,0,1,2,0)$; 5) $f_{1465}(5)=x_{1} \vee x_{2} x_{4} \vee x_{2} x_{5} \vee x_{3} x_{4} \vee x_{3} x_{5} \vee$ $\left.x_{4} x_{5}(0,1,5,0,0,0) ; 6\right) f_{1466}(5)=x_{1} x_{2} x_{3} \vee x_{1} x_{2} x_{4} \vee x_{1} x_{2} x_{5} \vee$ $\left.x_{1} x_{3} x_{4} \vee x_{1} x_{3} x_{5} \vee x_{2} x_{3} x_{4} x_{5}(0,0,0,5,1,0) ; 7\right) f_{1467}(5)=x_{1} x_{2} \vee$ $\left.x_{1} x_{3} \vee x_{1} x_{4} \vee x_{1} x_{5} \vee x_{2} x_{3} \vee x_{2} x_{4} x_{5} \vee x_{3} x_{4} x_{5}(0,0,5,2,0,0) ; 8\right)$ $f_{1468}(5)=x_{1} x_{2} \vee x_{1} x_{3} \vee x_{1} x_{4} x_{5} \vee x_{2} x_{3} x_{4} \vee x_{2} x_{3} x_{5} \vee x_{2} x_{4} x_{5} \vee$ $\left.x_{3} x_{4} x_{5}(0,0,2,5,0,0) ; 9\right) f_{1469}(5)=x_{1} x_{2} x_{3} \vee x_{1} x_{2} x_{4} \vee x_{1} x_{2} x_{5} \vee$ $\left.x_{1} x_{3} x_{4} \vee x_{1} x_{3} x_{5} \vee x_{1} x_{4} x_{5} \vee x_{2} x_{3} x_{4} \vee x_{2} x_{3} x_{5}(0,0,0,8,0,0) ; 10\right)$ $f_{1470}(5)=x_{1} x_{4} \vee x_{1} x_{5} \vee x_{2} x_{3} \vee x_{2} x_{4} \vee x_{2} x_{5} \vee x_{3} x_{4} \vee x_{3} x_{5} \vee x_{4} x_{5}$ $(0,0,8,0,0,0) ; 11) f_{1471}(5)=x_{2} x_{4} x_{5} \vee x_{3} x_{4} x_{5} \vee x_{1} x_{2} x_{3} x_{4} \vee$ $x_{1} x_{2} x_{3} x_{5}(0,0,0,2,2,0)$; 12) $f_{1472}(5)=x_{4} \vee x_{5} \vee x_{1} x_{2} \vee x_{1} x_{3}$ $\left.(0,2,2,0,0,0) ; 13) f_{1473}(5)=x_{1} x_{4} x_{5} \vee x_{2} x_{3} x_{4} x_{5}(0,0,0,1,1,0) ; 14\right)$ $f_{1474}(5)=x_{1} \vee x_{2} x_{3}(0,1,1,0,0,0)$. The fifth group contains $420 \mathrm{MBFs} f_{1461}(5) \ldots f_{1880}(5)$. The sixth group consists of 30 blocks $4.91 \ldots 4.120$. In the block 4.91 we have: 1) $f_{1881}(5)$ $=x_{1} x_{2} \vee x_{2} x_{3} \vee x_{3} x_{4}(0,0,3,0,0,0)$; 2) $f_{1882}(5)=x_{1} x_{2} x_{5} \vee x_{1} x_{4} x_{5}$ $\left.\vee x_{3} x_{4} x_{5}(0,0,0,3,0,0) ; 3\right) f_{1883}(5)=x_{5} \vee \vee x_{1} x_{3} \vee x_{1} x_{4} \vee x_{2} x_{4}$ $(0,1,3,0,0,0)$; 4) $f_{1884}(5)=x_{1} x_{3} x_{5} \vee x_{2} x_{3} x_{5} \vee x_{2} x_{4} x_{5} \vee$ $x_{1} x_{2} x_{3} x_{4} \quad(0,0,0,3,1,0)$; 5) $f_{1885}(5)=x_{1} x_{2} \vee x_{1} x_{5} \vee x_{2} x_{3} \vee x_{2} x_{5}$ $\left.\vee x_{3} x_{4} \vee x_{3} x_{5} \vee x_{4} x_{5} \quad(0,0,7,0,0,0) ; \quad 6\right) f_{1886}(5)=x_{1} x_{2} x_{3} \vee$ $x_{1} x_{2} x_{4} \vee x_{1} x_{2} x_{5} \vee x_{1} x_{3} x_{4} \vee x_{1} x_{4} x_{5} \vee x_{2} x_{3} x_{4} \vee x_{3} x_{4} x_{5}$ $(0,0,0,7,0,0) ; \quad 7) f_{1887}(5)=x_{1} x_{3} \vee x_{1} x_{4} \vee x_{2} x_{4} \vee x_{1} x_{2} x_{5} \vee$ $\left.x_{2} x_{3} x_{5} \vee x_{3} x_{4} x_{5}(0,0,3,3,0,0) ; 8\right) f_{1888}(5)=x_{1} x_{2} \vee x_{1} x_{4} \vee x_{3} x_{4} \vee$ $\left.x_{1} x_{3} x_{5} \vee x_{2} x_{3} x_{5} \vee x_{2} x_{4} x_{5}(0,0,3,3,0,0) ; 9\right) f_{1889}(5)=x_{1} x_{2} x_{3} \vee$ $x_{1} x_{2} x_{4} \vee x_{1} x_{3} x_{4} \vee x_{1} x_{3} x_{5} \vee x_{1} x_{4} x_{5} \vee x_{2} x_{3} x_{4} \vee x_{2} x_{4} x_{5}(0,0,0,7,0,0)$; 10) $f_{1890}(5)=x_{1} x_{3} \vee x_{1} x_{5} \vee x_{2} x_{3} \vee x_{2} x_{4} \vee x_{2} x_{5} \vee x_{3} x_{5} \vee x_{4} x_{5}$ $(0,0,7,0,0,0)$; 11) $f_{1891}(5)=x_{1} x_{2} x_{5} \vee x_{2} x_{3} x_{5} \vee x_{3} x_{4} x_{5} \vee x_{1} x_{2} x_{3} x_{4}$ $(0,0,0,3,1,0) ; 12) f_{1892}(5)=x_{5} \vee x_{1} x_{2} \vee x_{1} x_{4} \vee x_{3} x_{4}$ $(0,1,3,0,0,0) ; \quad 13) \quad f_{1893}(5)=x_{1} x_{3} x_{5} \vee x_{1} x_{4} x_{5} \vee x_{2} x_{4} x_{5}$ $(0,0,0,3,0,0)$; 14) $f_{1894}(5)=x_{1} x_{3} \vee x_{2} x_{3} \vee x_{2} x_{4}(0,0,3,0,0,0)$. The sixth group contains $420 \mathrm{MBFs} f_{1881}(5) \ldots f_{2300}(5)$. The seventh group consists of 30 blocks $4.121 \ldots 4.150$. In the block 4.121 we have: 1) $f_{2301}(5)=x_{1} \vee x_{2} x_{3} \vee x_{2} x_{4}$ $(0,1,2,0,0,0)$; 2) $f_{2302}(5)=x_{1} x_{3} x_{5} \vee x_{1} x_{4} x_{5} \vee x_{2} x_{3} x_{4} x_{5}$ $(0,0,0,2,1,0)$; 3) $f_{2303}(5)=x_{5} \vee x_{1} x_{2} \vee x_{1} x_{3} \vee x_{1} x_{4} \vee x_{3} x_{4}$ $(0,1,4,0,0,0)$; 4) $f_{2304}(5)=x_{1} x_{2} x_{5} \vee x_{1} x_{3} x_{5} \vee x_{2} x_{4} x_{5} \vee x_{3} x_{4} x_{5} \vee$ $x_{1} x_{2} x_{3} x_{4} \quad(0,0,0,4,1,0)$; 5) $f_{2305}(5)=x_{1} x_{5} \vee x_{2} x_{3} \vee x_{2} x_{4} \vee x_{2} x_{5}$ $\left.\vee x_{3} x_{5} \vee x_{4} x_{5} \vee x_{1} x_{3} x_{4} \quad(0,0,6,1,0,0) ; 6\right) f_{2306}(5)=x_{2} x_{5} \vee x_{1} x_{2} x_{3}$ $\left.\vee x_{1} x_{2} x_{4} \vee x_{1} x_{3} x_{4} \vee x_{1} x_{3} x_{5} \vee x_{1} x_{4} x_{5} \vee x_{2} x_{3} x_{4} \quad(0,0,1,6,0,0) ; \quad 7\right)$ $f_{2307}(5)=x_{1} x_{2} \vee x_{1} x_{3} x_{5} \vee x_{1} x_{4} x_{5} \vee x_{2} x_{3} x_{4} \vee x_{2} x_{3} x_{5} \vee x_{2} x_{4} x_{5} \vee$ $\left.x_{3} x_{4} x_{5}(0,0,1,6,0,0) ; 8\right) f_{2308}(5)=x_{1} x_{2} \vee x_{1} x_{3} \vee x_{1} x_{4} \vee x_{1} x_{5} \vee$ 
$\left.x_{2} x_{3} \vee x_{2} x_{4} \vee x_{3} x_{4} x_{5}(0,0,6,1,0,0) ; 9\right) f_{2309}(5)=x_{1} x_{2} x_{3} \vee x_{1} x_{2} x_{4} \vee$ $\left.x_{1} x_{2} x_{5} \vee x_{1} x_{3} x_{4} \vee x_{2} x_{3} x_{4} x_{5}(0,0,0,4,1,0) ; 10\right) f_{2310}(5)=x_{1} \vee x_{2} x_{5}$ $\left.\vee x_{3} x_{4} \vee x_{3} x_{5} \vee x_{4} x_{5}(0,1,4,0,0,0) ; 11\right) f_{2311}(5)=x_{1} x_{3} x_{5} \vee x_{1} x_{4} x_{5}$ $\left.\vee x_{1} x_{2} x_{3} x_{4} \quad(0,0,0,2,1,0) ; 12\right) f_{2312}(5)=x_{5} \vee x_{2} x_{3} \vee x_{2} x_{4}$ $(0,1,2,0,0,0)$; 13) $\left.f_{2313}(5)=x_{2} x_{5} \vee x_{3} x_{4} x_{5}(0,0,1,1,0,0) ; 14\right)$ $f_{1894}(5)=x_{1} x_{2} \vee x_{1} x_{3} x_{4}(0,0,1,1,0,0)$. The seventh group contains 420 MBFs $f_{2301}(5) \ldots f_{2720}(5)$. The eighth group consists of 30 blocks $4.151 \ldots 4.180$. In the block 4.151 we have: 1) $f_{2721}(5)=x_{1} x_{2} \vee x_{1} x_{3} \vee x_{1} x_{4} \vee x_{2} x_{3} \vee x_{2} x_{4}$ $(0,0,5,0,0,0) ; \quad$ 2) $f_{2722}(5)=x_{1} x_{3} x_{5} \vee x_{1} x_{4} x_{5} \vee x_{2} x_{3} x_{5} \vee x_{2} x_{4} x_{5}$ $\left.\vee x_{3} x_{4} x_{5}(0,0,0,5,0,0) ; 3\right) f_{2723}(5)=x_{5} \vee x_{3} x_{4} \vee x_{1} x_{2} x_{3} \vee x_{1} x_{2} x_{4}$ $(0,1,1,2,0,0)$; 4) $f_{2724}(5)=x_{3} x_{5} \vee x_{4} x_{5} \vee x_{1} x_{2} x_{5} \vee x_{1} x_{2} x_{3} x_{4}$ $(0,0,2,1,1,0)$; 5) $f_{2725}(5)=x_{1} x_{5} \vee x_{2} x_{5} \vee x_{3} x_{5} \vee x_{4} x_{5} \vee x_{1} x_{3} x_{4} \vee$ $x_{2} x_{3} x_{4}(0,0,4,2,0,0)$; 6) $f_{2726}(5)=x_{1} x_{5} \vee x_{2} x_{5} \vee x_{1} x_{2} x_{3} \vee$ $\left.x_{1} x_{2} x_{4} \vee x_{1} x_{3} x_{4} \vee x_{2} x_{3} x_{4}(0,0,2,4,0,0) ; 7\right) f_{2727}(5)=x_{1} x_{2} \vee$ $\left.x_{1} x_{3} x_{5} \vee x_{1} x_{4} x_{5} \vee x_{2} x_{3} x_{5} \vee x_{2} x_{4} x_{5} \vee x_{3} x_{4} x_{5} \quad(0,0,1,5,0,0) ; 8\right)$ $f_{2728}(5)=x_{1} x_{2} \vee x_{1} x_{3} \vee x_{1} x_{4} \vee x_{2} x_{3} \vee x_{2} x_{4} \vee x_{3} x_{4} x_{5}(0,0,5,1,0,0)$; 9) $f_{2729}(5)=x_{1} x_{2} x_{3} \vee x_{1} x_{2} x_{4} \vee x_{1} x_{2} x_{5} \vee x_{1} x_{3} x_{4} \vee x_{2} x_{3} x_{4}$ $(0,0,0,5,0,0)$; 10) $f_{2730}(5)=x_{1} x_{5} \vee x_{2} x_{5} \vee x_{3} x_{4} \vee x_{3} x_{5} \vee x_{4} x_{5}$ $(0,0,5,0,0,0) ; 11) f_{2731}(5)=x_{3} x_{5} \vee x_{4} x_{5} \vee x_{1} x_{2} x_{3} x_{4}$ $(0,0,2,0,1,0)$; 12) $f_{2732}(5)=x_{5} \vee x_{1} x_{2} x_{3} \vee x_{1} x_{2} x_{4}(0,1,0,2,0,0)$; 13) $f_{2733}(5)=x_{1} x_{5} \vee x_{2} x_{5} \vee x_{3} x_{4} x_{5}(0,0,2,1,0,0)$; 14) $f_{1734}(5)=$ $x_{1} x_{2} \vee x_{1} x_{3} x_{4} \vee x_{2} x_{3} x_{4} \quad(0,0,1,2,0,0)$. The eighth group contains 420 MBFs $f_{2721}(5) \ldots f_{3140}(5)$. The ninth group consists of 30 blocks $4.181 \ldots 4.210$. In the block 4.181 we have: 1) $\left.f_{3141}(5)=x_{1} x_{2} \vee x_{1} x_{3} x_{1} x_{4} x_{5} \vee x_{2} x_{3} x_{4}(0,0,2,2,0,0) ; 2\right)$ $\left.f_{3142}(5)=x_{1} x_{5} \vee x_{2} x_{3} \vee x_{2} x_{4} x_{5} \vee x_{3} x_{4} x_{5} \quad(0,0,2,2,0,0) ; 3\right) f_{3143}(5)$ $\left.=x_{2} x_{5} \vee x_{3} x_{5} \vee x_{1} x_{2} x_{3} \vee x_{1} x_{2} x_{4} \vee x_{1} x_{3} x_{4}(0,0,2,3,0,0) ; 4\right) f_{3144}(5)$ $\left.=x_{2} x_{5} \vee x_{3} x_{5} \vee x_{4} x_{5} \vee x_{1} x_{2} x_{4} \vee x_{1} x_{3} x_{4}(0,0,3,2,0,0) ; 5\right) f_{3145}(5)$ $=x_{1} x_{5} \vee x_{4} x_{5} \vee x_{2} x_{3} x_{4} \vee x_{2} x_{3} x_{5}(0,0,2,2,0,0)$; 6) $f_{3146}(5)=$ $\left.x_{1} x_{4} \vee x_{1} x_{5} \vee x_{1} x_{2} x_{3} \vee x_{2} x_{3} x_{4}(0,0,2,2,0,0) ; 7\right) f_{3147}(5)=x_{1} x_{2} \vee$ $\left.x_{1} x_{3} \vee x_{1} x_{4} \vee x_{2} x_{4} x_{5} \vee x_{3} x_{4} x_{5}(0,0,3,2,0,0) ; 8\right) f_{3148}(5)=x_{1} x_{2} \vee$ $\left.x_{1} x_{3} \vee x_{2} x_{3} x_{5} \vee x_{2} x_{4} x_{5} \vee x_{3} x_{4} x_{5} \quad(0,0,2,3,0,0) ; \quad 9\right) \quad f_{3149}(5)$ $=x_{1} x_{5} \vee x_{2} x_{3} \vee x_{1} x_{2} x_{4} \vee x_{1} x_{3} x_{4}(0,0,2,2,0,0)$; 10) $f_{3150}(5)=$ $\left.x_{2} x_{5} \vee x_{3} x_{5} \vee x_{1} x_{4} x_{5} \vee x_{2} x_{3} x_{4}(0,0,2,2,0,0) ; 11\right) f_{3151}(5)=x_{2} x_{5} \vee$ $\left.x_{3} x_{5} \vee x_{4} x_{5} \vee x_{1} x_{2} x_{3} \vee x_{2} x_{3} x_{4}(0,0,3,2,0,0) ; \quad 12\right) f_{3152}(5)=x_{1} x_{5}$ $\left.\vee x_{4} x_{5} \vee x_{1} x_{2} x_{3} \vee x_{1} x_{2} x_{4} \vee x_{1} x_{3} x_{4} \quad(0,0,2,3,0,0) ; 13\right)$ $\left.f_{3153}(5)=x_{1} x_{4} \vee x_{1} x_{5} \vee x_{2} x_{3} x_{5} \vee x_{2} x_{4} x_{5} \vee x_{3} x_{4} x_{5}(0,0,2,3,0,0) ; 14\right)$ $f_{3154}(5)=x_{1} x_{2} \vee x_{1} x_{3} \vee x_{1} x_{4} \vee x_{2} x_{3} x_{4} \vee x_{2} x_{3} x_{5}$ $(0,0,3,2,0,0)$.The ninth group contains 420 MBFs $f_{3141}(5) \ldots f_{3560}(5)$. The tenth group consists of 60 blocks $4.211 \ldots 4.270$. In the block 4.211 we have: 1) $f_{3561}(5)=$ $x_{1} x_{2} \vee x_{1} x_{3} x_{1} x_{4} \vee x_{2} x_{3}(0,0,4,0,0,0) ; \quad$ 2) $f_{3562}(5)=x_{1} x_{4} x_{5} \vee$ $\left.x_{2} x_{3} x_{5} \vee x_{2} x_{4} x_{5} \vee x_{3} x_{4} x_{5}(0,0,0,4,0,0) ; 3\right) f_{3563}(5)=x_{5} \vee x_{2} x_{4} \vee$ $\vee x_{3} x_{4} \vee x_{1} x_{2} x_{3}(0,1,2,1,0,0)$; 4) $f_{3564}(5)=x_{4} x_{5} \vee x_{1} x_{2} x_{5} \vee$ $x_{1} x_{3} x_{5} \vee x_{1} x_{2} x_{3} x_{4}(0,0,1,2,1,0)$; 5) $f_{3565}(5)=x_{1} x_{4} \vee x_{1} x_{5} \vee x_{2} x_{5} \vee$ $x_{3} x_{5} \vee x_{4} x_{5} \vee x_{2} x_{3} x_{4}(0,0,5,1,0,0)$; 6) $f_{3566}(5)=x_{1} x_{5} \vee x_{1} x_{2} x_{3}$ $\left.\vee x_{1} x_{2} x_{4} \vee x_{1} x_{3} x_{4} \vee x_{2} x_{3} x_{4} \vee x_{2} x_{3} x_{5}(0,0,1,5,0,0) ; 7\right) f_{3567}(5)=$ $\left.x_{1} x_{2} \vee x_{1} x_{3} \vee x_{1} x_{4} x_{5} \vee x_{2} x_{3} x_{5} \vee x_{2} x_{4} x_{5} \vee x_{3} x_{4} x_{5}(0,0,2,4,0,0) ; 8\right)$ $f_{3568}(5)=x_{1} x_{2} \vee x_{1} x_{3} \vee x_{1} x_{4} \vee x_{2} x_{3} \vee x_{2} x_{4} x_{5} \vee x_{3} x_{4} x_{5}$ $(0,0,4,2,0,0)$; 9) $f_{3569}(5)=x_{1} x_{2} x_{3} \vee x_{1} x_{2} x_{4} \vee x_{1} x_{2} x_{5} \vee$ $\left.x_{1} x_{3} x_{4} \vee x_{1} x_{3} x_{5} \vee x_{2} x_{3} x_{4}(0,0,0,6,0,0) ; 10\right) f_{3570}(5)=x_{1} x_{5} \vee x_{2} x_{4}$ $\left.\vee x_{2} x_{5} \vee x_{3} x_{4} \vee x_{3} x_{5} \vee x_{4} x_{5}(0,0,6,0,0,0) ; 11\right) f_{3571}(5)=x_{4} x_{5} \vee$ $x_{2} x_{3} x_{5} \vee x_{1} x_{2} x_{3} x_{4}(0,0,1,1,1,0)$; 12) $f_{3572}(5)=x_{5} \vee x_{1} x_{4} \vee$ $x_{1} x_{2} x_{3}(0,1,1,1,0,0)$; 13) $f_{3573}(5)=x_{1} x_{5} \vee x_{2} x_{4} x_{5} \vee x_{3} x_{4} x_{5}$ $(0,0,1,2,0,0)$; 14) $f_{3574}(5)=x_{1} x_{2} \vee x_{1} x_{3} \vee x_{2} x_{3} x_{4} \quad(0,0,2,1,0,0)$.
The tenth group contains $840 \mathrm{MBFs} f_{3561}(5) \ldots f_{4400}(5)$. The eleventh group consists of 60 blocks $4.271 \ldots 4.330$. In the block 4.271 we have: 1) $f_{4401}(5)=x_{1} x_{2} \vee x_{2} x_{3} x_{3} x_{4} \vee x_{3} x_{5}$ $(0,0,4,0,0,0)$; 2) $f_{4402}(5)=x_{1} x_{2} x_{4} \vee x_{1} x_{2} x_{5} \vee x_{1} x_{4} x_{5} \vee x_{3} x_{4} x_{5}$ $(0,0,0,4,0,0)$; 3) $f_{4403}(5)=x_{1} x_{3} \vee x_{1} x_{4} \vee x_{1} x_{5} \vee x_{2} x_{4} \vee x_{2} x_{5} \vee$ $x_{4} x_{5}(0,0,6,0,0,0)$; 4) $f_{4404}(5)=x_{1} x_{2} x_{3} \vee x_{1} x_{3} x_{4} \vee x_{1} x_{3} x_{5} \vee$ $\left.x_{2} x_{3} x_{4} \vee x_{2} x_{3} x_{5} \vee x_{2} x_{4} x_{5}(0,0,0,6,0,0) ; 5\right) f_{4405}(5)=x_{1} x_{2} \vee x_{2} x_{3}$ $\left.\vee x_{3} x_{4} \vee x_{3} x_{5} \vee x_{1} x_{4} x_{5} \vee x_{2} x_{4} x_{5}(0,0,4,2,0,0) ; 6\right) f_{4406}(5)=x_{1} x_{3}$ $\left.\vee x_{2} x_{3} \vee x_{1} x_{2} x_{4} \vee x_{1} x_{2} x_{5} \vee x_{1} x_{4} x_{5} \vee x_{3} x_{4} x_{5}(0,0,2,4,0,0) ; 7\right)$ $f_{4407}(5)=x_{1} x_{3} \vee x_{1} x_{2} x_{4} \vee x_{1} x_{2} x_{5} \vee x_{2} x_{3} x_{4} \vee x_{2} x_{3} x_{5} \vee x_{3} x_{4} x_{5}$ $(0,0,1,5,0,0)$; 8) $f_{4408}(5)=x_{1} x_{2} \vee x_{1} x_{4} \vee x_{1} x_{5} \vee x_{3} x_{4} \vee x_{3} x_{5} \vee$ $\left.x_{2} x_{4} x_{5}(0,0,5,1,0,0) ; 9\right) f_{4409}(5)=x_{1} x_{2} x_{3} \vee x_{1} x_{3} x_{4} \vee x_{1} x_{3} x_{5} \vee$ $\left.x_{1} x_{4} x_{5} \vee x_{2} x_{4} x_{5}(0,0,0,5,0,0) ; 10\right) f_{4410}(5)=x_{1} x_{3} \vee x_{2} x_{3} \vee x_{2} x_{4} \vee$ $\left.x_{2} x_{5} \vee x_{4} x_{5} \quad(0,0,5,0,0,0) ; 11\right) f_{4411}(5)=x_{1} x_{2} x_{4} \vee x_{1} x_{2} x_{5} \vee$ $\left.x_{2} x_{3} x_{4} \vee x_{2} x_{3} x_{5} \vee x_{3} x_{4} x_{5} \quad(0,0,0,5,0,0) ; 12\right) f_{4412}(5)=x_{1} x_{2} \vee$ $\left.x_{1} x_{4} \vee x_{1} x_{5} \vee x_{3} x_{4} \vee x_{3} x_{5}(0,0,5,0,0,0) ; 13\right) f_{4413}(5)=x_{1} x_{3} \vee$ $\left.x_{1} x_{4} x_{5} \vee x_{2} x_{4} x_{5}(0,0,1,2,0,0) ; 14\right) f_{4414}(5)=x_{1} x_{3} \vee x_{2} x_{3} \vee x_{2} x_{4} x_{5}$ $(0,0,2,1,0,0)$. The eleventh group contains $840 \mathrm{MBFs}$ $f_{4401}(5) \ldots f_{5240}(5)$. The twelfth group consists of 60 blocks $4.331 \ldots 4.390$. In the block 4.331 we have: 1) $f_{5241}(5)=$ $\left.x_{1} x_{3} \vee x_{1} x_{4} \vee x_{2} x_{3} \vee x_{3} x_{4} x_{5}(0,0,3,1,0,0) ; 2\right) f_{5242}(5)=x_{1} x_{2} \vee$ $\left.x_{1} x_{4} x_{5} \vee x_{2} x_{3} x_{5} \vee x_{2} x_{4} x_{5}(0,0,1,3,0,0) ; 3\right) f_{5243}(5)=x_{1} x_{2} \vee x_{1} x_{5} \vee$ $x_{2} x_{4} \vee x_{2} x_{5} \vee x_{1} x_{3} x_{4}(0,0,4,1,0,0)$; 4) $f_{5244}(5)=x_{2} x_{5} \vee x_{1} x_{3} x_{4} \vee$ $\left.x_{1} x_{3} x_{5} \vee x_{2} x_{3} x_{4} \vee x_{3} x_{4} x_{5}(0,0,1,4,0,0) ; 5\right) f_{5245}(5)=x_{2} x_{3} \vee x_{3} x_{5}$ $\left.\vee x_{4} x_{5} \vee x_{1} x_{2} x_{4} \vee x_{1} x_{2} x_{5}(0,0,3,2,0,0) ; 6\right) f_{5246}(5)=x_{3} x_{4} \vee x_{3} x_{5}$ $\left.\vee x_{1} x_{2} x_{3} \vee x_{1} x_{2} x_{4} \vee x_{1} x_{4} x_{5}(0,0,2,3,0,0) ; 7\right) f_{5247}(5)=x_{1} x_{3} \vee x_{3} x_{4}$ $\left.\vee x_{1} x_{4} x_{5} \vee x_{2} x_{3} x_{5} \vee x_{2} x_{4} x_{5}(0,0,2,3,0,0) ; \quad 8\right) f_{5248}(5)=x_{1} x_{3}$ $\left.\vee x_{1} x_{4} \vee x_{2} x_{3} \vee x_{1} x_{2} x_{5} \vee x_{2} x_{4} x_{5}(0,0,3,2,0,0) ; 9\right) f_{5249}(5)=x_{1} x_{2}$ $\left.\vee x_{1} x_{3} x_{4} \vee x_{1} x_{3} x_{5} \vee x_{2} x_{3} x_{4} \vee x_{3} x_{4} x_{5}(0,0,1,4,0,0) ; 10\right) f_{5250}(5)=$ $x_{1} x_{2} \vee x_{1} x_{5} \vee x_{2} x_{4} \vee x_{2} x_{5} \vee x_{3} x_{4} x_{5}(0,0,4,1,0,0)$; 11) $f_{5251}(5)$ $=x_{2} x_{5} \vee x_{1} x_{2} x_{3} \vee x_{1} x_{2} x_{4} \vee x_{1} x_{4} x_{5} \quad(0,0,1,3,0,0)$; 12) $f_{5252}(5)=$ $\left.x_{2} x_{3} \vee x_{3} x_{5} \vee x_{4} x_{5} \vee x_{1} x_{3} x_{4}(0,0,3,1,0,0) ; 13\right) f_{5253}(5)=x_{3} x_{4} \vee$ $x_{3} x_{5} \vee x_{1} x_{2} x_{5} \vee x_{2} x_{4} x_{5}(0,0,2,2,0,0)$; 14) $f_{5254}(5)=x_{1} x_{3} \vee x_{3} x_{4}$ $\vee x_{1} x_{2} x_{4} \vee x_{1} x_{2} x_{5}(0,0,2,2,0,0)$. The twelfth group contains $840 \mathrm{MBFs} f_{5241}(5) \ldots f_{6080}(5)$. Only 390 blocks of power 14 contain 5460 MBFs.

On fig. 4 block 5.1 power of 32 MBFs of rank 5 is shown.

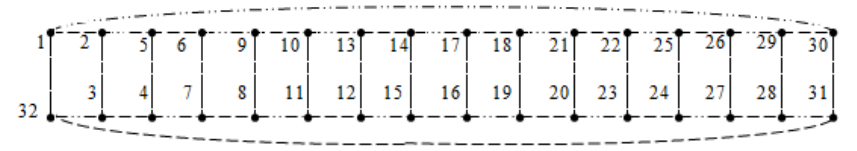

Figure 4. Block 5.1 power of $32 \mathrm{MBFs}$ of rank 5

In block 5.1 we have: 1) $f_{6081}(5)=x_{1} x_{2} \vee x_{2} x_{3} \vee x_{3} x_{4} \vee x_{4} x_{5}$ $(0,0,4,0,0,0) ; 2) \quad f_{6082}(5)=x_{1} x_{2} x_{3} \vee x_{1} x_{2} x_{5} \vee x_{1} x_{4} x_{5} \vee x_{3} x_{4} x_{5}$ $(0,0,0,4,0,0)$; 3) $f_{6083}(5)=x_{1} x_{3} \vee x_{1} x_{4} \vee x_{1} x_{5} \vee x_{2} x_{4} \vee x_{2} x_{5} \vee$ $x_{3} x_{5}(0,0,6,0,0,0)$; 4) $f_{6084}(5)=x_{1} x_{2} x_{4} \vee x_{1} x_{3} x_{4} \vee x_{1} x_{3} x_{5} \vee$ $\left.x_{2} x_{3} x_{4} \vee x_{2} x_{3} x_{5} \vee x_{2} x_{4} x_{5}(0,0,0,6,0,0) ; 5\right) f_{6085}(5)=x_{1} x_{2} \vee x_{2} x_{3} \vee$ $x_{3} x_{4} \vee x_{4} x_{5} \vee x_{1} x_{3} x_{5}(0,0,4,1,0,0)$; 6) $f_{6086}(5)=x_{2} x_{4} \vee x_{1} x_{2} x_{3} \vee$ $\left.x_{1} x_{2} x_{5} \vee x_{1} x_{4} x_{5} \vee x_{3} x_{4} x_{5}(0,0,1,4,0,0) ; 7\right) f_{6087}(5)=x_{1} x_{4} \vee x_{2} x_{4}$ $\left.\vee x_{2} x_{5} \vee x_{1} x_{2} x_{3} \vee x_{3} x_{4} x_{5}(0,0,3,2,0,0) ; 8\right) f_{6088}(5)=x_{1} x_{2} \vee$ $x_{4} x_{5} \vee x_{1} x_{3} x_{4} \vee \mathrm{x}_{1} \mathrm{x}_{3} \mathrm{x}_{5} \vee x_{2} x_{3} x_{5}(0,0,2,3,0,0)$; 9) $f_{6089}(5)=x_{1} x_{5}$ $\left.\vee x_{1} x_{2} x_{4} \vee x_{1} x_{3} x_{4} \vee x_{2} x_{3} x_{4} \vee x_{2} x_{3} x_{5} \vee x_{2} x_{4} x_{5} \quad(0,0,1,5,0,0) ; 10\right)$ $f_{6090}(5)=x_{1} x_{3} \vee x_{1} x_{4} \vee x_{1} x_{5} \vee x_{2} x_{5} \vee x_{3} x_{5} \vee x_{2} x_{3} x_{4}(0,0,5,1,0,0)$; 
11) $f_{6091}(5)=x_{1} x_{2} x_{3} \vee x_{1} x_{2} x_{5} \vee x_{1} x_{3} x_{5} \vee x_{1} x_{4} x_{5} \vee x_{3} x_{4} x_{5}$ $(0,0,0,5,0,0)$; 12) $f_{6092}(5)=x_{1} x_{2} \vee x_{2} x_{3} \vee x_{2} x_{4} \vee x_{3} x_{4} \vee x_{4} x_{5}$ $(0,0,5,0,0,0) ; 13) f_{6093}(5)=x_{2} x_{4} \vee x_{1} x_{3} x_{4} \vee x_{2} x_{3} x_{5}(0,0,1,2,0,0)$; 14) $f_{6094}(5)=x_{1} x_{4} \vee x_{2} x_{5} \vee x_{1} x_{3} x_{5} \quad(0,0,2,1,0,0)$; 15) $f_{6095}(5)=$ $\left.x_{1} x_{2} \vee x_{1} x_{5} \vee x_{4} x_{5} \vee x_{2} x_{3} x_{4}(0,0,3,1,0,0) ; 16\right) f_{6096}(5)=x_{1} x_{5} \vee$ $x_{1} x_{2} x_{3} \vee x_{2} x_{3} x_{4} \vee x_{3} x_{4} x_{5} \quad(0,0,1,3,0,0)$; 17) $f_{6097}(5)=x_{1} x_{3} \vee$ $x_{1} x_{4} \vee x_{2} x_{5} \vee x_{3} x_{5}(0,0,4,0,0,0)$; 18) $f_{6098}(5)=x_{1} x_{2} x_{4} \vee x_{1} x_{3} x_{4} \vee$ $x_{2} x_{3} x_{5} \vee x_{2} x_{4} x_{5}(0,0,0,4,0,0)$; 19) $f_{6099}(5)=x_{1} x_{3} \vee x_{1} x_{4} \vee x_{1} x_{5} \vee$ $\left.x_{2} x_{4} \vee x_{2} x_{5} \vee x_{3} x_{5}(0,0,6,0,0,0) ; 20\right) f_{6100}(5)=x_{1} x_{2} x_{3} \vee x_{1} x_{2} x_{5} \vee$ $\left.x_{1} x_{3} x_{5} \vee x_{1} x_{4} x_{5} \vee x_{2} x_{3} x_{4} \vee x_{3} x_{4} x_{5}(0,0,0,6,0,0) ; 21\right) f_{6101}(5)=$ $\left.x_{1} x_{3} \vee x_{1} x_{4} \vee x_{2} x_{5} \vee x_{3} x_{5} \vee x_{2} x_{3} x_{4}(0,0,4,1,0,0) ; 22\right) f_{6102}(5)=$ $\left.x_{1} x_{5} \vee x_{1} x_{2} x_{4} \vee x_{1} x_{3} x_{4} \vee x_{2} x_{3} x_{5} \vee x_{2} x_{4} x_{5}(0,0,1,4,0,0) ; 23\right)$ $\left.f_{6103}(5)=x_{1} x_{2} \vee x_{1} x_{5} \vee x_{4} x_{5} \vee x_{1} x_{3} x_{4} \vee x_{2} x_{3} x_{5} \quad(0,0,3,2,0,0) ; 24\right)$ $\left.f_{6104}(5)=x_{1} x_{4} x_{2} x_{5} \vee x_{1} x_{2} x_{3} \vee x_{2} x_{3} x_{4} \vee x_{3} x_{4} x_{5}(0,0,2,3,0,0) ; 25\right)$ $f_{6105}(5)=x_{2} x_{4} \cdot x_{1} x_{2} x_{3} \vee x_{1} x_{2} x_{5} \vee x_{1} x_{3} x_{5} \vee x_{1} x_{4} x_{5} \vee$ $\left.x_{3} x_{4} x_{5}(0,0,1,5,0,0) ; 26\right) f_{6106}(5)=x_{1} x_{2} \vee x_{2} x_{3} \vee x_{2} x_{4} \vee x_{3} x_{4} \vee$ $\left.x_{4} x_{5} \vee x_{1} x_{3} x_{5}(0,0,5,1,0,0) ; 27\right) f_{6107}(5)=x_{1} x_{2} x_{4} \vee x_{1} x_{3} x_{4} \vee$ $\left.x_{2} x_{3} x_{4} \vee x_{2} x_{3} x_{5} \vee x_{2} x_{4} x_{5}(0,0,0,5,0,0) ; 28\right) f_{6108}(5)==x_{1} x_{3} \vee$ $x_{1} x_{4} \vee x_{1} x_{5} \vee x_{2} x_{5} \vee x_{3} x_{5}(0,0,5,0,0,0)$; 29) $f_{6109}(5)=x_{1} x_{5} \vee$ $\left.x_{1} x_{2} x_{3} \vee x_{3} x_{4} x_{5}(0,0,1,2,0,0) ; 30\right) \mathrm{f}_{6110}(5)=x_{1} x_{2} \vee x_{4} x_{5} \vee$ $\left.x_{2} x_{3} x_{4} \quad(0,0,2,1,0,0) ; 31\right) f_{6111}(5)=x_{1} x_{4} \vee x_{2} x_{4} \vee x_{2} x_{5} \vee x_{1} x_{3} x_{5}$ $(0,0,3,1,0,0)$; 32) $f_{6112}(5)=x_{2} x_{4} \vee x_{1} x_{3} x_{4} \vee x_{1} x_{3} x_{5} \vee x_{2} x_{3} x_{5}$ $(0,0,1,3,0,0)$. In total for MBFs 5 of a rank there are 30 isomorphic blocks of power 32. They contain $960 \mathrm{MBFs}$ $f_{6081}(5) \ldots f_{7040}(5)$.

On fig. 5 block 6.1 power of 54 MBFs of rank 5 is shown

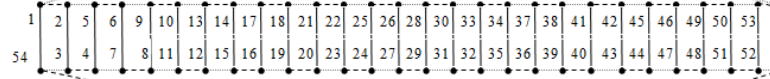

Figure 5. Block 6.1 power of $54 \mathrm{MBFs}$ of rank 5

In block 6.1 we have: 1) $f_{7041}(5)=x_{1} x_{3} \vee x_{1} x_{4} \vee x_{2} x_{5} \vee$ $x_{3} x_{4} x_{5}(0,0,3,1,0,0)$; 2) $f_{7042}(5)=x_{1} x_{2} \vee x_{1} x_{3} x_{4} \vee x_{2} x_{3} x_{5} \vee$ $x_{2} x_{4} x_{5}(0,0,1,3,0,0)$; 3) $f_{7043}(5)=x_{1} x_{2} \vee x_{1} x_{5} \vee x_{2} x_{3} \vee x_{2} x_{4} \vee$ $x_{1} x_{3} x_{4}(0,0,4,1,0,0)$; 4) $f_{7044}(5)=x_{2} x_{5} \vee x_{1} x_{3} \mathrm{x}_{5} \vee x_{1} x_{4} x_{5} \vee$ $\left.x_{2} x_{3} x_{4} \vee x_{3} x_{4} x_{5} \quad(0,0,1,4,0,0) ; 5\right) f_{7045}(5)=x_{2} x_{5} \vee x_{3} x_{5} \vee x_{4} x_{5}$ $\left.\vee \vee x_{1} x_{2} x_{3} \vee x_{1} x_{2} x_{4} \vee x_{2} x_{3} x_{4}(0,0,3,3,0,0) ; 6\right) f_{7046}(5)=x_{1} x_{5}$ $\left.\vee x_{3} x_{5} \vee x_{4} x_{5} \vee x_{1} x_{2} x_{3} \vee x_{1} x_{2} x_{4} \vee x_{1} x_{3} x_{4} \quad(0,0,3,3,0,0) ; 7\right)$ $f_{7047}(5)=x_{1} x_{5} \vee x_{1} x_{3} x_{4} \vee x_{2} x_{3} x_{5} \vee x_{2} x_{4} x_{5} \vee x_{3} x_{4} x_{5}(0,0,1,4,0,0)$; 8) $f_{7048}(5)=x_{1} x_{2} \vee x_{1} x_{3} \vee x_{1} x_{4} \vee x_{2} x_{5} \vee x_{2} x_{3} x_{4}(0,0,4,1,0,0)$; 9) $f_{7049}(5)=x_{1} x_{2} \vee x_{1} x_{3} x_{5} \vee x_{1} x_{4} x_{5} \vee x_{2} x_{3} x_{4} \quad(0,0,1,3,0,0)$; 10) $\left.f_{7050}(5)=x_{1} x_{5} \vee x_{2} x_{3} \vee x_{2} x_{4} \vee x_{3} x_{4} x_{5}(0,0,3,1,0,0) ; 11\right)$ $f_{7051}(5)=x_{2} x_{5} \vee x_{1} x_{2} x_{3} \vee x_{1} x_{2} x_{4} \vee x_{1} x_{3} x_{4} \vee x_{3} x_{4} x_{5}$ $(0,0,1,4,0,0) ; \quad 12) \quad f_{7052}(5)=x_{1} x_{2} \vee x_{2} x_{5} \vee x_{3} x_{5} \vee x_{4} x_{5}$ $\vee x_{1} x_{3} x_{4}(0,0,4,1,0,0)$; 13) $f_{7053}(5)=x_{1} x_{5} \vee x_{2} x_{3} x_{4} \vee$ $x_{2} x_{3} x_{5} \vee x_{2} x_{4} x_{5}(0,0,1,3,0,0)$; 14) $f_{7054}(5)=x_{1} x_{3} \vee x_{1} x_{4} \vee x_{1} x_{5}$ $\vee x_{2} x_{3} x_{4}(0,0,3,1,0,0)$; 15) $f_{7055}(5)=x_{1} x_{2} \vee x_{1} x_{3} \vee x_{1} x_{4} \vee$ $x_{3} x_{4} x_{5}(0,0,3,1,0,0)$; 16) $f_{7056}(5)=x_{1} x_{2} \vee x_{2} x_{3} x_{5} \vee x_{2} x_{4} x_{5} \vee$ $x_{3} x_{4} x_{5}(0,0,1,3,0,0)$; 17) $f_{7057}(5)=x_{1} x_{5} \vee x_{2} x_{3} \vee x_{2} x_{4} \vee x_{2} x_{5}$ $\left.\vee x_{1} x_{3} x_{4}(0,0,4,1,0,0) ; 18\right) f_{7058}(5)=x_{2} x_{5} \vee x_{1} x_{3} x_{4} \vee x_{1} x_{3} x_{5} \vee$ $\left.x_{1} x_{4} x_{5} \vee x_{2} x_{3} x_{4}(0,0,1,4,0,0) ; 19\right) \mathrm{f}_{7059}(5)=x_{1} x_{2} \vee x_{3} x_{5} \vee x_{4} x_{5}$ $\left.\vee x_{2} x_{3} x_{4}(0,0,3,1,0,0) ; 20\right) f_{7060}(5)=x_{1} x_{5} \vee x_{1} x_{2} x_{3} \vee x_{1} x_{2} x_{4} \vee$ $\left.x_{3} x_{4} x_{5}(0,0,1,3,0,0) ; 21\right) f_{7061}(5)=x_{1} x_{3} \vee x_{1} x_{4} \vee x_{1} x_{5} \vee x_{2} x_{5} \vee$ $x_{3} x_{4} x_{5}(0,0,4,1,0,0)$; 22) $f_{7062}(5)=x_{1} x_{2} \vee x_{1} x_{3} x_{4} \vee x_{2} x_{3} x_{4} \vee$ $\left.x_{2} x_{3} x_{5} \vee x_{2} x_{4} x_{5}(0,0,1,4,0,0) ; 23\right) f_{7063}(5)=x_{1} x_{2} \vee x_{2} x_{3} \vee$ $\left.x_{2} x_{4} \vee x_{1} x_{3} x_{4} \vee x_{1} x_{3} x_{5} \vee x_{1} x_{4} x_{5}(0,0,3,3,0,0) ; 24\right) f_{7064}(5)=$ $\left.x_{2} x_{3} \vee x_{2} x_{4} \vee x_{2} x_{5} \vee x_{1} x_{3} x_{5} \vee x_{1} x_{4} x_{5} \vee x_{3} x_{4} x_{5}(0,0,3,3,0,0) ; 25\right)$ $f_{7065}(5)=x_{2} x_{5} \vee x_{1} x_{2} x_{3} \vee x_{1} x_{2} x_{4} \vee x_{2} x_{3} x_{4} \vee x_{3} x_{4} x_{5}$ $(0,0,1,4,0,0) ; 26) f_{7066}(5)=x_{1} x_{2} \vee x_{1} x_{5} \vee x_{3} x_{5} \vee x_{4} x_{5} \vee$ $x_{1} x_{3} x_{4}(0,0,4,1,0,0)$; 27) $f_{7067}(5)=x_{1} x_{5} \vee x_{1} x_{3} x_{4} \vee x_{2} x_{3} x_{5} \vee$ $\left.x_{2} x_{4} x_{5}(0,0,1,3,0,0) ; 28\right) f_{7068}(5)=x_{1} x_{3} \vee x_{1} x_{4} \vee x_{2} x_{5} \vee x_{2} x_{3} x_{4}$ $(0,0,3,1,0,0) ; 29) f_{7069}(5)=x_{1} x_{2} \vee x_{1} x_{3} x_{5} \vee x_{1} x_{4} x_{5} \vee x_{2} x_{3} x_{4} \vee$ $x_{3} x_{4} x_{5}(0,0,1,4,0,0)$; 30) $f_{7070}(5)=x_{1} x_{2} \vee x_{1} x_{5} \vee x_{2} x_{3} \vee x_{2} x_{4} \vee$ $\left.x_{3} x_{4} x_{5}(0,0,4,1,0,0) ; 31\right) f_{7071}(5)=x_{2} x_{5} \vee x_{1} x_{2} x_{3} \vee x_{1} x_{2} x_{4} \vee$ $x_{1} x_{3} x_{4}(0,0,1,3,0,0)$; 32) $f_{7072}(5)=x_{2} x_{5} \vee x_{3} x_{5} \vee x_{4} x_{5} \vee x_{1} x_{3} x_{4}$ $(0,0,3,1,0,0) ; 33) f_{7073}(5)=x_{1} x_{5} \vee x_{3} x_{5} \vee x_{4} x_{5} \vee x_{2} x_{3} x_{4}$ $(0,0,3,1,0,0)$; 34) $f_{7074}(5)=x_{1} x_{5} \vee x_{1} x_{2} x_{3} \vee x_{1} x_{2} x_{4} \vee x_{2} x_{3} x_{4}$ $(0,0,1,3,0,0)$; 35) $f_{7075}(5)=x_{1} x_{2} \vee x_{1} x_{3} \vee x_{1} x_{4} \vee x_{2} x_{5} \vee x_{3} x_{4} x_{5}$ $(0,0,4,1,0,0)$; 36) $f_{7076}(5)=x_{1} x_{2} \vee x_{1} x_{3} x_{4} \vee x_{2} x_{3} x_{5} \vee x_{2} x_{4} x_{5} \vee$ $x_{3} x_{4} x_{5}(0,0,1,4,0,0)$; 37) $f_{7077}(5)=x_{1} x_{5} \vee x_{2} x_{3} \vee x_{2} x_{4} \vee$ $\left.x_{1} x_{3} x_{4} \quad(0,0,3,1,0,0) ; 38\right) f_{7078}(5)=x_{2} x_{5} \vee x_{1} x_{3} x_{5} \vee x_{1} x_{4} x_{5} \vee$ $\left.x_{2} x_{3} x_{4}(0,0,1,3,0,0) ; 39\right) f_{7079}(5)=x_{1} x_{2} \vee x_{2} x_{5} \vee x_{3} x_{5} \vee x_{4} x_{5} \vee$ $x_{2} x_{3} x_{4}(0,0,4,1,0,0)$; 40) $f_{7080}(5)=x_{1} x_{5} \vee x_{1} x_{2} x_{3} \vee x_{1} x_{2} x_{4} \vee$ $\left.x_{1} x_{3} x_{4} \vee x_{3} x_{4} x_{5}(0,0,1,4,0,0) ; 41\right) f_{7081}(5)=x_{1} x_{3} \vee x_{1} x_{4} \vee x_{1} x_{5}$ $\left.\vee x_{2} x_{3} x_{5} \vee x_{2} x_{4} x_{5} \vee x_{3} x_{4} x_{5}(0,0,3,3,0,0) ; 42\right) f_{7082}(5)=x_{1} x_{2} \vee$ $\left.x_{1} x_{3} \vee x_{1} x_{4} \vee x_{2} x_{3} x_{4} \vee x_{2} x_{3} x_{5} \vee x_{2} x_{4} x_{5} \quad(0,0,3,3,0,0) ; 43\right)$ $f_{7083}(5)=x_{1} x_{2} \vee x_{1} x_{3} x_{4} \vee x_{1} x_{3} x_{5} \vee x_{1} x_{4} x_{5} \vee x_{2} x_{3} x_{4}$ $(0,0,1,4,0,0)$; 44) $f_{7084}(5)=x_{1} x_{5} \vee x_{2} x_{3} \vee x_{2} x_{4} \vee x_{2} x_{5} \vee$ $\left.x_{3} x_{4} x_{5}(0,0,4,1,0,0) ; 45\right) f_{7085}(5)=x_{2} x_{5} \vee x_{1} x_{2} x_{3} \vee x_{1} x_{2} x_{4} \vee$ $\left.x_{3} x_{4} x_{5}(0,0,1,3,0,0) ; 46\right) f_{7086}(5)=x_{1} x_{2} \vee x_{3} x_{5} \vee x_{4} x_{5} \vee$ $\left.x_{1} x_{3} x_{4}(0,0,3,1,0,0) ; 47\right) f_{7087}(5)=x_{1} x_{5} \vee x_{1} x_{3} x_{4} \vee x_{2} x_{3} x_{4} \vee$ $x_{2} x_{3} x_{5} \vee x_{2} x_{4} x_{5}(0,0,1,4,0,0)$; 48) $f_{7088}(5)=x_{1} x_{3} \vee x_{1} x_{4} \vee x_{1} x_{5}$ $\vee x_{2} x_{5} \vee x_{2} x_{3} x_{4}(0,0,4,1,0,0)$; 49) $f_{7089}(5)=x_{1} x_{2} \vee x_{1} x_{3} x_{5} \vee$ $x_{1} x_{4} x_{5} \vee x_{3} x_{4} x_{5}(0,0,1,3,0,0)$; 50) $f_{7090}(5)=x_{1} x_{2} \vee x_{2} x_{3} \vee x_{2} x_{4}$ $\vee x_{3} x_{4} x_{5}(0,0,3,1,0,0)$; 51) $f_{7091}(5)=x_{2} x_{3} \vee x_{2} x_{4} \vee x_{2} x_{5} \vee$ $x_{1} x_{3} x_{4}(0,0,3,1,0,0)$; 52) $f_{7092}(5)=x_{2} x_{5} \vee x_{1} x_{3} x_{4} \vee x_{1} x_{3} x_{5} \vee$ $x_{1} x_{4} x_{5}(0,0,1,3,0,0)$; 53) $f_{7093}(5)=x_{1} x_{2} \vee x_{1} x_{5} \vee x_{3} x_{5} \vee x_{4} x_{5} \vee$ $x_{2} x_{3} x_{4}(0,0,4,1,0,0)$; 54) $f_{7094}(5)=x_{1} x_{5} \vee x_{1} x_{2} x_{3} \vee x_{1} x_{2} x_{4} \vee$ $x_{2} x_{3} x_{4} \vee x_{3} x_{4} x_{5}(0,0,1,4,0,0)$. In total for MBFs of rank 5 there are 10 isomorphic blocks of power 54. They contain $540 \mathrm{MBFs} f_{7041}(5) \ldots f_{7580}(5)$.

Thus, 7581 MBFs of a rank 5 contains in 6 groups of similar blocks or in 23 groups of isomorphic blocks or in 522 blocks. From 7581 MBFs 276 have a maximal type. In 5 figures in article 6 blocks ( 1 block from each group of similar blocks) are represented. For complete research of all the $7581 \mathrm{MBFs}$ it is enough to study 23 blocks (1 block from each group of isomorphic blocks). These 23 block contain 313 MBFs and all of them are presented in this paper. Such reduction considerably accelerates search of the necessary MBF in cases where necessary their exhaustive search. With the growth of the rank is the acceleration increases. To set 23 blocks is enough to set 23 MBFs (1 MBF simplest of each block). To obtain the rest of MBFs unit is enough to consistently apply to a given $\mathrm{MBF}$ operations, disjunctive complement and duality.

Any permutation on the set of 5 variables $x_{1}, x_{2}, x_{3}, x_{4}, x_{5}$ transfers a given MBF or isomorphic MBF, or into itself. In total there are $5 !=120$ permutations of which form a symmetric group $\mathrm{S}_{5}$. Permutations which transfer MBF $f$ of rank 5 to itself form a subgroup of $G_{f}$ of $S_{5}$ group which is 
called as the MBF $f$ stabilizer. The elements of the quotient set $D_{f}=S_{5} / G_{f}$ transfers MBF $f$ to isomorphic MBF. The quotient set $D_{f}$ consists of $120 / k$ elements, where $k$ is the number of elements in the stabilizer $G_{f}$.

For each block $\mathrm{B}$ of 14 groups of isomorphic blocks for any MBF of block $B$ we have $G_{f}=G_{B}$, ie group stabilizer MBF $f$ and block $B$ are the same. For each block $B$ of 14 groups of isomorphic blocks (7 groups contain blocks of 7 MBF and 7 groups contain blocks of $14 \mathrm{MBF}$ ) we have any two MBF of block $B$ is nonisomorphic.

For each block $B$ of 7 groups of isomorphic blocks (this include 5 groups with blocks of $14 \mathrm{MBF}, 1$ group with blocks of $4 \mathrm{MBF}$ and 1 group with blocks of $32 \mathrm{MBF}$ ) and for any $\operatorname{MBF} f_{i}(5)$ of block $B$ stabilizer $G_{B}$ is 2 times more of stabilizer $G_{f}$. For each block $B$ of these 7 groups of isomorphic blocks are for any MBF of block $B$ this block contains 2 isomorphic to it MBF (including herself).

For each block B of 2 groups of isomorphic blocks (includes 1 group with blocks of $6 \mathrm{MBFs}$ and 1 group with blocks of $54 \mathrm{MBFs}$ ) and for any MBF $f_{i}(5)$ of block $B$ we have stabilizer $G_{B} 6$ times more of stabilizer $G_{f}$. For each block $B$ of these two groups isomorphic blocks we have for any MBF block $B$ this block contains 6 isomorphic to it MBF (including herself).

Taking into account these properties it is easy to make a count of all nonequivalent MBFs of rank 5 .

$\mathrm{R}(5)=7 * 7+7 * 14+(5 * 14+4+32) / 2+(6+54) /$ $6=49+98+106 / 2+60 / 6=147+53+10=210$.

We show how using blocks, can find some special types of MBF.

We shall call MBF completely unsymmetric, if the stabilizer group consists of a single identity permutation. In this case, MBF of rank 5 has $5 !=120$ isomorphic functions. Of the above properties of blocks follows that completely unsymmetric are the only MBF of 21 group of isomorphic blocks. Therefore, there are $14 * 60=840 \mathrm{MBF}$ of 5 rank. Of these $840 \mathrm{MBF}$ can select only 7 mutually nonisomorphic.

We shall call MBF self-dual if it is dual to itself. Such $\mathrm{MBF}$ is represented in the image of the block by a point in a triangle, from which does not come out line corresponding to the operation of duality. Such MBF of rank 5 can only be in groups of isomorphic blocks 1 to 7 . Thus the number of self-dual MBF of rank 5 equals $1+5+5+10+10+20+$ $30=81$. Of these $81 \mathrm{MBF}$ can only select 7 are mutually nonisomorphic. Similarly, the number of self-complementary disjunctive and number of conjunctive self-complementary MBF of rank 5 also equals 81 . Of these, can also select to 7 are mutually nonisomorphic.

In our case, the stabilizer $G_{f}$ MBF $f$ contained in the group of automorphisms $G_{B}$ (stabilizer) of block $B$, which belongs the $\operatorname{MBF} f$. In the general case, $G_{B}$ is not a normal subgroup of $S_{5}$, but splits the $S_{5}$ into cosets. All elements of the coset $S_{5}$ for $G_{B}$ transferred block $B$ to the same isomorphic block him, or in himself. In the latter case each MBF of the block $B$ is transformed either into itself or isomorphic $\mathrm{MBF}$ belonging to the same block.
Permutations of the group $S_{5}$ can be conveniently represented in the form of vectors, or cycles, consisting of indexes of their constituent variables. Permutation in the form vector $(5,3,2,1,4)$ means that the variable $x_{1}$ becomes variable $x_{5}$, variable $x_{2}$ becomes variable $x_{3}$ and so on. All vector components must be different. The same permutation in the form cycles is as follows: $(1,5,4)(2.3)$. Unit group $S_{5}$ in the form of a vector is recorded as $(1,2,3,4,5)$ and as cycles as 1 .

For complete enumeration of MBFs of rank 5 it is necessary to find for each of 23 groups of isomorphic blocks partitioning into cosets $S_{5}$ on $G_{B}$. Select in each coset of one element and regulate these elements. For ordering can be considered as vectors of permutations, corresponding to the chosen elements, numbers, and the components of the vectors digits of these numbers. In this case, all items will be sorted in ascending order of numbers.

For block 6.1 the group of automorphisms of $G_{B}$ consists of 12 elements. Next, each element of the $G_{B}$ is shown as a vector and as cycles: 1$)(1,2,3,4,5)=1 ; 2)(1,2,4,3,5)=(3,4)$; 3) $(1,5,3,4,2)=(2,5)$; 4) $(1,5,4,3,2)=(2,5)(3,4)$; 5) $(2,1,3,4,5)=(1,2) ; 6)(2,1,4,3,5)=(1,2)(3,4) ; 7)(2,5,3,4,1)$ $=(1,2,5)$; 8) $(2,5,4,3,1)=(1,2,5)(3,4)$; 9) $(5,1,3,4,2)=$ $(1,5,2) ; 10(5,1,4,3,2)=(1,5,2)(3,4) 11)(5,2,3,4,1)=(1,5)$; $12(5,2,4,3,1)=(1,5)(3,4)$. Selected items ten cosets S5 on $G_{B}$ have the form: 1) $\left.\left.(1,2,3,4,5)=1 ; 2\right)(1,2,3,5,4)=(4,5) ; 3\right)$ $(1,2,5,4,3)=(3,5)$; 4) $(1,3,2,4,5)=(2,3) ; 5)(1,3,2,5,4)=$ $(2,3)(4,5) ; 6)(1,4,3,2,5)=(2,4) ; 7)(3,2,1,4,5)=(1,3) ; 8)$ $(3,2,1,5,4)=(1,3)(4,5) ; 9)(3,4,1,2,5)=(1,3)(2,4) ; 10)$ $(4,2,3,1,5)=(1,4)$. These elements are ordered. Using elements of $2 \ldots 10$ easy of MBFs block 6.1 to obtain MBFs blocks $6.2 \ldots 6.10$. For example, from MBFs $f_{7041}(5)=$ $x_{1} x_{3} \vee x_{1} x_{4} \vee x_{2} x_{5} \vee x_{3} x_{4} x_{5}$ of the block 6.1 we obtain the following MBFs: 6.2) $f_{7095}(5)=x_{1} x_{3} \vee x_{1} x_{5} \vee x_{2} x_{4} \vee x_{3} x_{4} x_{5}$; 6.3) $f_{7149}(5)=x_{1} x_{4} \vee x_{1} x_{5} \vee x_{2} x_{3} \vee x_{3} x_{4} x_{5}$; 6.4) $f_{7203}(5)=x_{1} x_{2}$ $\left.\vee \mathrm{x}_{1} \mathrm{x}_{4} \vee x_{2} x_{3} \vee x_{2} x_{4} x_{5} ; 6.5\right) f_{7257}(5)=x_{1} x_{2} \vee x_{1} x_{5} \vee x_{3} x_{4} \vee$ $x_{2} x_{4} x_{5}$; 6.6) $\left.f_{7311}(5)=x_{1} x_{2} \vee x_{1} x_{3} \vee x_{4} x_{5} \vee x_{2} x_{3} x_{5} ; 6.7\right) f_{7365}(5)=$ $x_{1} x_{3} \vee x_{2} x_{5} \vee x_{3} x_{4} \vee x_{1} x_{4} x_{5}$; 6.8) $f_{7419}(5)=x_{1} x_{3} \vee x_{2} x_{4} \vee x_{3} x_{5}$ $\vee x_{1} x_{4} x_{5}$; 6.9) $f_{7473}(5)=x_{1} x_{3} \vee x_{2} x_{3} \vee x_{4} x_{5} \vee x_{1} x_{2} x_{5}$; 6.10) $f_{7527}(5)=x_{1} x_{4} \vee x_{2} x_{5} \vee x_{3} x_{4} \vee x_{1} x_{3} x_{5}$. Similarly, it is easy to obtain and the remaining MBFs blocks 6.2... 6.10.

For example, using this method, we can find the Dedekind number to 5 variables. Dedekind number for 5 variables is convenient to count through similar blocks. For the 5 variables, there are 6 groups of similar blocks. Of these, by one group of 4, 6, 7, 14, 32 and 54 MBFs, Groups of similar blocks of $4,6,32$ and 54 MBFs contain on 1 group isomorphic blocks. Group of similar blocks of 7 MBFs includes 7 groups of isomorphic blocks and group of similar blocks of $14 \mathrm{MBFs}$ contains 12 groups of isomorphic blocks. In groups of similar blocks of 4 and 6 MBFs contains 5 and 6 blocks. In the group of 7 similar blocks MBFs contains $1+2 \cdot 5+2 \cdot 10+1 \cdot 20+1 \cdot 30=81$ blocks. In the group of similar blocks of $14 \mathrm{MBFs}$ contains $1 \cdot 10+2 \cdot 15+1 \cdot 20+5 \cdot 30+3 \cdot 60=390$ blocks. In groups of similar blocks of 32 and 54 MBFs contains 30 and 10 blocks. Hence, the fifth Dedekind number 


$$
\begin{aligned}
& D(5)=4 \cdot 6+6 \cdot 5+7 \cdot 81+14 \cdot 390+30 \cdot 32+10 \cdot 54= \\
& =24+30+567+5460+960+540=7581
\end{aligned}
$$

\section{Conclusions}

In conclusion, we note the following. Research MBFs of rank 5 allowed us to study the properties of more than 7500 MBFs. The proposed methods of analysis and sorting of MBFs can be used for network analysis and construction of new codes for cryptosystems. MBFs blocks have a simpler structure than the lattice MBFs, so the method of construction of MBFs blocks significantly reduces the exhaustive search MBFs.

\section{REFERENCES}

[1] Dedekind R. Über Zerlegungen von Zahlen durch ihre grössten gemeinsamen Teiler. Festschrift Hoch. Braunschweig u. ges. Werke. II, 1897, 103-148.

[2] Wiedemann, Doug A computation of the eighth Dedekind number. Order 8 (1991), no. 1, 5-6.

[3] Pieter-Jan Hoedt, Parallelizing with MPI in Java to Find the ninth Dedekind Number, http://oeis.org/A000372, 2015.

[4] В.Г.Ткаченко, О.В.Синявский Построение корректирую щего кода для криптосистем на основе типов монотонных булевых функций. Наукові праці ОНАЗ ім. О.С. Попова, 2010, № 1, 85 - 92.
[5] Tkachenco V.G., Sinyavsky O.V. Construction of Cryptosystem on the Basis of Triangular Codes, Computer Science and Information Technology, 2 (2014), 300 - 307. DOI: $10.13189 /$ csit.2014.020703.

[6] Tkachenco V.G., Sinyavsky O.V., Construction of cryptosystem on the basis quadrangular codes. Nauka i studia, Przemysl, 2013, 18 - 27.

[7] Ткаченко В.Г. Классификация монотонных булевых функций при синтезе цифровых схем. Наукові праці ОНАЗ ім. О.С. Попова, 2008, № 1, $35-43$.

[8] Tkachenco V.G., Sinyavsky O.V. Recursive Construction of n-gonal Codes on the Basis of Block Design, Computer Science and Information Technology, 2 (2014), 331 - 338. DOI: $10.13189 /$ csit.2014.020803.

[9] Tkachenco V.G., Sinyavsky O.V. Blocks of Monotone Boolean Functions, Computer Science and Information Technology Vol. 4(2), pp. 72 - 78. DOI: 10.13189/csit.2016.040203.

[10] K. Engel, Sperner theory, Cambridge University Press, 1997.

[11] V. Bakoev, One more way for counting monotone boolean functions, in: Thirteenth International Workshop on Algebraic and Combinatorial Coding Theory, 2012, p. 4752.

[12] R. Fidytek, A. W. Mostowski, R. Somla, A. Szepietowski, Algorithms counting monotone boolean functions, Information Processing Letters 79 (2001) 203209.

[13] T. Stephen, T. Yusun, Counting inequivalent monotone boolean functions, Discrete Appl. Math. 167 (2014) 15-24. doi:10.1016/j.dam.2013.11.015. http://dx.doi.org/10.1016/j.dam.2013.11.015
URL 